\title{
Trading Volume, Price Autocorrelation and Volatility under Proportional Transaction Costs
}

\author{
Hua Cheng* \\ University of Paris Dauphine - Department of Economics
}

*SDF (Financial Strategies \& Dynamics), P313A, Department of Economics, University of Paris Dauphine, Place de Maréchal de Lattre de Tassigny. 75775. Paris Cedex 16. Tel: 0033 1440549 47. Email address: hua.cheng@dauphine.fr. 


\begin{abstract}
We develop a dynamic model in which traders have differential information about the true value of the risky asset and trade the risky asset with proportional transaction costs. We show that without additional assumption, trading volume can not totally remove the noise in the pricing equation. However, because trading volume increases in the absolute value of noisy per capita supply change, it provides useful information on the asset fundamental value which cannot be inferred from the equilibrium price.
\end{abstract}

We further investigate the relation between trading volume, price autocorrelation, return volatility and proportional transaction costs. Firstly, trading volume decreases in proportional transaction costs and the influence of proportional transaction costs decreases at the margin. Secondly, price autocorrelation can be generated by proportional transaction costs: under no transaction costs, the equilibrium prices at date 1 and 2 are not correlated; however under proportional transaction costs, they are correlated - the higher (lower) the equilibrium price at date 1 , the lower (higher) the equilibrium price at date 2. Thirdly, we show that return volatility may be increasing in proportional transaction costs, which is contrary to Stiglitz 1989, Summers \& Summers 1989's reasoning but is consistent with Umlauf 1993 and Jones \& Seguin 1997's empirical results.

Keywords: Trading Volume, Autocorrelation, Volatility

JEL codes: G12, G14, D82 
In the standard rational expectation equilibrium model with aggregate supply uncertainty, if trading volume plays any role, it is mainly to resolve the noisy supply from the equilibrium price equation. All traders observing trading volume know the aggregate supply and thus there is a revealing price. While it is very difficult for traders to submit their orders conditional on price and trading volume in practice, we investigate the role of trading volume in the equilibrium in which traders make their decisions conditional up to but not including the market statistic resulting from their desired trade ${ }^{1}$.

Transaction costs are an important factor in determining the trading behavior of market participants ${ }^{2}$. On the consequence, transactions costs should affect trading volume, asset prices and their time series features. While considerable attention has focused on the effects of transaction costs on asset prices, there are very few models which investigate their influences on price autocorrelation and return volatility ${ }^{3}$. Increased transaction costs are usually thought to reduce the incentive to traders and therefore produce a thinner market. Thin trading tends to induce or increase autocorrelation, as in Boudoukh, Richardson \& Whitelaw 1994. Stiglitz 1989 and Summers \& Summers 1989 argue that transaction costs should diminish return volatility. The reasoning underpinning this claim stems from the belief that noise trades, which are not based on information about underlying values, may move prices away from the intrinsic value and increase volatility ${ }^{4}$.

However, this reasoning is not consistent with the empirical results (Umlauf

\footnotetext{
${ }^{1}$ This approach is first suggested by Hellwig 1982. Blume \& Easley 1984 use this approach to examine the information content of past market prices. Blume, Easley \& O'Hara 1994 use it to investigate the role of volume. Cheng 2005a uses it to study the role of volume in an economy with proportional transaction costs and the influence of transaction costs in a static model.

${ }^{2}$ Transaction costs fall into two broad categories. First are the direct penuniary costs of trading. These include the market-maker's spread, the broker's fees, and any transaction taxes, such as stamp duties. Second are the indirect costs. These include the costs of acquiring and processing information about asset values, companies, market movements and any other information which may be relevant to the decision to buy and sell assets. We define transaction costs in a narrow way and they include only the first in our model.

3 The direction and magnitude of the affects of transaction costs on asset prices are still subject to considerable controversy and debate. Constantinides 1986, Heaton \& Lucas 1996, and Huang 2003 show that transaction costs have only a small impact on asset prices. Amihud \& Mendelson 1986 and Lo, Mamaysky \& Wang 2004 find that the liquidity discount of transaction costs can be substantial, despite relatively small transaction costs. While those models argue that there exists always the liquidity premium of transaction costs, Vayanos 1998 and Cheng 2005a suggest that asset price may increase in its transaction costs.

${ }^{4}$ They define noise traders broadly. This definition includes (but is not limited to) portfolio insurers and other so-called positive feedback traders, since their trades are based only on changes in reported prices rather than on intrinsic value, as well as others who believe (irrationally) that trading systems, horoscopes, etc., are beneficial in forecasting prices.
} 
1993, Jones \& Seguin 1997, Green, Maggioni \& Murinde 2000, and Hau 2006, etc.). In the end of their paper, Jones \& Seguin 1997 conclude that "Our results, when combined with those of Umlauf 1993, suggest that the logic of increasing transaction taxes to reduce the impact of noise traders and therefore, to reduce volatility, does not withstand empirical scrutiny. Indeed, our results indicate that increasing transaction costs through any avenue may well have an effect exactly opposite from that intended."

We develop a two periods model in which traders have differential information about the true value of the risky asset and there are proportional transaction costs on the risky asset. We show that without additional assumption, trading volume can not totally remove the noise in the pricing equation. However, because trading volume increases in the absolute value of noisy per capita supply change, it provides useful information on the asset fundamental value which cannot be inferred from the equilibrium price.

Our second result is that trading volume decreases in proportional transaction costs and the influence of proportional transaction costs decreases at the margin. The third result is about how price autocorrelation can be generated only by proportional transaction costs: under no transaction costs, the equilibrium prices at date 1 and 2 are not correlated (independent); however under proportional transaction costs, they are correlated - for all the other parameters given, the higher the equilibrium price (common information) at date 1, the lower the equilibrium price at date 2. Our fourth result is to show that return volatility may be increasing in proportional transaction costs, which is contrary to Stiglitz 1989, Summers \& Summers 1989's reasoning but is consistent with most empirical results (Umlauf 1993, Jones \& Seguin 1997, Green, Maggioni \& Murinde 2000, and Hau 2006, etc.).

The rest of the article is structured as follows: in section 1 we develop the basic model. In section 2 we study the equilibriums at date 1 and 2 and the relation between the noisy per capita supply change and trading volume. In section 3 we analyze the relationship between trading volume, price autocorrelation, return volatility and proportional transaction costs. In section 4 , we contrast our results to several relevant empirical papers and argue that our argument is consistent with the available evidence. We present conclusions in section 5 . 


\section{Economy}

We consider a simple economy with two assets in the economy: a riskless asset and a risky asset. The riskless asset is assumed to have an infinitely elastic supply and the supply of the risky asset is random. Let $x_{1}$ and $x_{2}$ denote the random per capita supplies of the risky asset at dates 1 and 2 , respectively ${ }^{5}$. The interest rate of riskless asset is simplified to be 0 and its price is normalized to one. All assets are traded in a competitive market.

There are two types of risk-averse traders in the economy and we divide the traders into two groups with $N_{I}=u N$ traders in group 1 and $N_{U}=(1-u) N$ traders in group 2. The two classes of investors are different in their information about the state of the economy which is further defined as follows. We focus on the results with $u N$ and $(1-u) N \rightarrow \infty$, namely the results in the large economy. $u$ is supposed to be time independent and informed (uninformed) traders at date 1 are still informed (uninformed) at date 2 .

\subsection{Preference}

All traders have constant absolute risk aversion (CARA). Each trader maximizes his expected utility of consumption at date 3

$$
E\left[U\left(w_{3, i}^{j}\right) \mid \digamma_{t, i}^{j}\right]=E\left[-\exp \left(-R w_{3}^{j}\right) \mid \digamma_{t, i}^{j}\right]
$$

where $w_{3, i}^{j}$ is trader $j$ in group $i$ 's wealth at date 3 , the common absolute risk aversion $R$ is simplified to 1 , and $\digamma_{t, i}^{j}$ is the information set available to trader $j$ in group $i$ at date $t$.

\section{$1.2 \quad$ Information Structure}

Traders are a priori identical: at date 0 , each trader enters the first period with an endowment of $z_{0}$ units of the riskless asset and has the identical beliefs about the payoff of the risky asset $\psi$, which is assumed to be a normal distribution $N\left(\psi_{0}, 1 / \rho_{0}\right)$.

Just prior to the opening of the market at date 1 and 2, each trader receives

\footnotetext{
${ }^{5}$ Uncertainty in the per capita supply reflect uncertain order flow based on noise traders. In our model, noise traders is norrowly defined and include only those who trade for liquidity reasons. Then it is logical that their trades are not (or little) influenced by transaction costs. Whether there is transaction cost or not at date 2, the noisy per capita supply is distributed in the same way.
} 
his private signal and then traders trade with each other. The private signals that each group receives are identically distributed but the distributions for two groups are different. More precisely

$$
\begin{aligned}
& y_{t, i}^{j}=\psi+w_{t}+e_{t}^{j} \text { for informed trader } j \text { in group } 1 \\
& y_{t, i}^{j}=\psi+w_{t}+\varepsilon_{t}^{j} \text { for uninformed trader } j \text { in group } 2
\end{aligned}, t=1,2
$$

where $w_{t}$ is a common error term distributed $N\left(0,1 / \rho_{t}^{w}\right), e_{t}^{j}$ and $\varepsilon_{t}^{j}$ are idiosyncratic errors for traders in group 1 and group 2 which are distributed $N\left(0,1 / \rho_{t}^{1}\right)$ and $N\left(0,1 / \rho_{t}^{2}\right)$, respectively. Assume further that $\rho_{t}^{1}>\rho_{t}^{2}$. Since the information that traders in group 1 receive is more reliable than that traders in group 2 receive, we refer to traders in group 1 as informed traders and traders in group 2 as uninformed traders. At the end of the second period (at date 3 ), the value of the risky asset is revealed and all traders consume their wealth.

To simplify the notation, we write each trader's information structures of the risky asset after receiving the private signal in the following way

$$
\begin{aligned}
& y_{t, i}^{j} \sim N\left(\psi, 1 / \rho_{t}^{s 1}\right), \text { for } \text { informed trader } j \text { in group } 1 \\
& y_{t, i}^{j} \sim N\left(\psi, 1 / \rho_{t}^{s 2}\right), \text { for uninformed trader } j \text { in group } 2
\end{aligned}
$$

where $\rho_{t}^{s 1}=\rho_{t}^{1} \rho_{t}^{w} /\left(\rho_{t}^{1}+\rho_{t}^{w}\right)$ and $\rho_{t}^{s 2}=\rho_{t}^{2} \rho_{t}^{w} /\left(\rho_{t}^{2}+\rho_{t}^{w}\right)$. Conditional on $w_{t}$

$$
\begin{aligned}
& y_{t}^{j} \mid w_{t} \sim N\left(\theta_{t}, 1 / \rho_{t}^{1}\right), \text { for informed trader } j \text { in group } 1 \\
& y_{t}^{j} \mid w_{t} \sim N\left(\theta_{t}, 1 / \rho_{t}^{2}\right), \text { for uninformed trader } j \text { in group } 2
\end{aligned}
$$

where $\theta_{t}=\psi+w_{t}$ denotes common information to all traders at date $t$. So by the Strong Law of Large Numbers, the mean signal in each group, $\bar{y}_{t}^{1}$ and $\bar{y}_{t}^{2}$ converges almost surely to $\theta_{t}$ as $N \rightarrow \infty$. In the large economy, the mean signal is almost surely equal to the ture value plus the common error. All the random variables $\left(w_{t}, e_{t}^{j}, \varepsilon_{t}^{j}\right.$, and $\left.x_{t}\right)$ are supposed to be mutually independent.

\subsection{Optimization}

Ideally, each trader would predict the stochastic process of prices conditional on his information set, and solve the intertemporal decision problem and take potential capital gains into account. However, this problem is tractable if and only if future prices are normally distributed. It is not the case in our model for two reasons. Firstly, the precision of the common and private information 
are random and we don't know their future value. Secondly, even though we suppose that the precision of the common and private information are constant or determinant, because of transaction costs, the equilibrium price in the second period will never be normally distributed.

Following Brown \& Jenning 1989 and Blume, Easley \& O'Hara 1994, we assume that traders have myopic, or naive, demands so that each trader chooses his demand to maximize his expected utility at date 3 without taking possible future transactions into account. We suppose that each trader makes his decision conditional on all information up to but not including the market statistics from their desired trades (the equilibrium price and trading volume). Then the information sets of each trader are

$$
\begin{aligned}
\digamma_{1, i}^{j} & =\left\{\psi_{0}, y_{1, i}^{j}, \operatorname{sign}\left(x_{1}\right)\right\} \text { at date } 1 \\
\digamma_{2, i}^{j} & =\left\{\psi_{0}, y_{1, i}^{j}, p_{1}, V_{1}, \operatorname{sign}\left(x_{1}\right), y_{2, i}^{j}, \operatorname{sign}\left(x_{2}-x_{1}\right)\right\} \text { at date } 2
\end{aligned}
$$

The reason why we suppose the signs of the noisy per capita supply (change) are known to all traders is explained in the beginning of subsection 2.2. This assumption does not affect the equilibrium in the first period but does affect that in the second period.

\subsection{Transaction Costs}

To examine the effect of transaction costs on the equilibrium price and trading volume, we introduce a per-share fee, $c$, for each share bought or sold of the risky asset in the second period. Thus the total transaction costs increase with the number of shares traded ${ }^{6} 7$. This is consistent with most theoretical models, e.g., Vayanos 1998, Barron \& Karpoff 2004, and empirical evidence, e.g.,Brennan \& Chordia 1993.

\footnotetext{
${ }^{6}$ Including a transaction cost at date 1 complicates our analysis, but we do not expect that it affects our main results.

${ }^{7}$ Our results require that the total transaction costs are not decreasing with the number of shares traded and that they are the same for the same shares bought or sold. For example, the retults retain if transaction costs consist only of a fixed, or lump-sum, component. However, if we suppose that for each transaction, the buyer and seller have to pay a combined exoggenous fixed cost and the allocation of this fixed cost between buyer and sell is determined endogenously as in Lo, Mamaysky \& Wang 2004, the results change.
} 


\subsection{Market Clearing}

We can investigate the market clearing in a "stock" sense and in a "flow" sense. The market for risky asset clears in a "stock" sense if total holdings at a given point in time are equal to the noisy per capita supply. The market for risky asset clears in a "flow" sense if the net number of shares bought and sold is equal to the change of the noisy per capita supply. In the first period, these two senses of market clearing are the same. However, although we can also compute the equilibrium price in either sense in the second period, it is relatively more convenient to compute the equilibrium price in a "stock" sense because of price autocorrelation investigated in section 3.

\section{Equilibrium}

A feasible trading strategy requires that planned asset holdings be measurable with respect to the trader's available information set and satisfy the individual's budget at each trading date. Let $d_{1, i}^{j}$ denote trader $j$ in group $i$ 's time 1 holding of the risky asset. Then the payoff at date 3 is $d_{1, i}^{j} \psi+z_{0}-d_{1, i}^{j} p_{1}$ at date 1 and $d_{2, i}^{j} \psi+d_{1, i}^{j} p_{2}+z_{0}-d_{1, i}^{j} p_{1}-d_{2, i}^{j} p_{2}-\left|d_{2, i}^{j}-d_{1, i}^{j}\right| c$ at date 2 . The optimal trading strategy is determined by solving

$$
J_{1, i}\left(d_{1, i}^{j}\right)=\max _{d_{1, i}^{j}}\left[-\exp \left(-\left(d_{1, i}^{j} \psi+z_{0}-d_{1, i}^{j} p_{1}\right)\right) \mid \digamma_{1, i}^{j}\right] \text { at date } 1
$$

and

$$
\begin{aligned}
J_{2, i}\left(d_{2, i}^{j}\right)= & \max _{d_{2, i}^{j}}\left[-\exp \left(-\left(z_{0}+d_{2, i}^{j}\left(\psi-p_{2}\right)\right.\right.\right. \\
& \left.\left.\left.+d_{1, i}^{j}\left(p_{2}-p_{1}\right)-\left|d_{2, i}^{j}-d_{1, i}^{j}\right| c\right)\right) \mid \digamma_{2, i}^{j}\right] \text { at date } 2
\end{aligned}
$$

An equilibrium is a pair of demand functions $\left(d_{1, i}^{j}, d_{2, i}^{j}\right)$ for each trader and a pair of the equilibrium price function $\left(p_{1}, p_{2}\right)$ that together satisfy the following conditions. First, the equilibrium price $p_{t}$ are functions of $\digamma_{t}$ through their dependence on traders' demands and the noisy per capita supplies. Second, each trader's strategy is feasible and solves above optimization equation. Finally, traders' strategies and the equilibrium prices are such that market clears. The market-clearing condition in a "stock" or "flow" senses are written respectively 
as

$$
\begin{gathered}
d_{t}=\frac{1}{N} \sum_{j=1}^{N} d_{t, i}^{j}=x_{t} \text { in a "stock" sensee } \\
d_{t}-d_{t-1}=\frac{1}{N} \sum_{j=1}^{N}\left(d_{t, i}^{j}-d_{t-1, i}^{j}\right)=x_{t}-x_{t-1} \text { in a "flow" sense }
\end{gathered}
$$

We use per capital trading volume as a measure of trading volume ${ }^{8}$

$$
V_{t}=\frac{1}{2 N}\left(\sum_{j=1}^{u N}\left|d_{t, 1}^{j}\right|+\sum_{j=u N+1}^{N}\left|d_{t, 2}^{j}\right|\right)+\frac{\left|x_{t}-x_{t-1}\right|}{2}
$$

\subsection{Equilibrium in the First Period}

Proposition 1 In the first period without transaction costs,

(1) the equilibrium price is

$$
p_{1}=\frac{\rho_{0} \psi_{0}+\left(u \rho_{1}^{s 1}+(1-u) \rho_{1}^{s 2}\right) \theta_{1}-x_{1}}{\rho_{0}+u \rho_{1}^{s 1}+(1-u) \rho_{1}^{s 2}}
$$

(2) the demand of each trader is

$$
d_{1}\left(y_{1, i}^{j}\right)=\rho_{0}\left(\psi_{0}-p_{1}\right)+\rho_{1}^{s i}\left(y_{1, i}^{j}-p_{1}\right)
$$

(3) and, given $\theta_{1}$ and $x_{1}$, trading volume is

$$
V=\frac{u}{2} V_{1}^{1}+\frac{(1-u)}{2} V_{1}^{2}+\frac{\left|x_{1}\right|}{2}
$$

where

$$
\begin{gathered}
V_{1}^{i}=2 \frac{\rho_{1}^{s i}}{\sqrt{\rho_{1}^{i}}} \phi\left(\frac{\delta_{1}^{i} \sqrt{\rho_{1}^{i}}}{\rho_{1}^{s i}}\right)-\delta_{1}^{i} \Phi\left(-\frac{\delta_{1}^{i} \sqrt{\rho_{1}^{i}}}{\rho_{1}^{s i}}\right)+\delta_{1}^{i} \Phi\left(\frac{\delta_{1}^{i} \sqrt{\rho_{1}^{i}}}{\rho_{1}^{s i}}\right) \\
\delta_{1}^{i}=\rho_{0}\left(\psi_{0}-p_{1}\right)+\rho_{1}^{s i}\left(\theta_{1}-p_{1}\right)
\end{gathered}
$$

\footnotetext{
${ }^{8} \mathrm{~A}$ number of measures of volume have been proposed and studied: aggregate share volume, individual share volume, aggregate dollar volume, individual dollar volume, individual turnover, aggregate turnover, etc. See Lo \& Wang 2000 for an excellent analysis on these different measures.
} 
where $\phi$ is the standard normal density, and $\Phi$ is the standard normal cumulative distribution function ${ }^{9}$.

Three remarks are in order. Firstly, the demand of each trader does not depend on the noisy per capita supply directly and depends on it only indirectly via the equilibrium price. Secondly, for the same common information, the higher the noisy per capita supply, the lower the equilibrium price. Because the noisy per capita supply is the counterpart of the noisy per capita demand of liquidity traders, the high positive per capita supply means high sell order from liquidity traders. This sell pressure pushes down the equilibrium price. For the same reason, the high negative per capita supply pushs up the equilibrium price. Thirdly, because neither informed nor uninformed trader knows this noisy per capita supply, neither of them knows the common information from only the equilibrium price. For example, if the equilibrium price is equal to traders' identical prior expectation $\psi_{0}$, it may be due to a positive common information with a positive per capita noisy supply, or a negative common information with a negative per capita noisy supply, or a neutral common information with zero noisy per capita supply. Both of them now need look at trading volume.

Proposition 2 In the first period without transaction costs, for the equilibrium price given, trading volume is a decreasing function of the noisy per capita supply when $x_{1}<0$ and an increasing function of noisy per capita supply when $x_{1}>0$ and achieves its minimum at $x_{1}=0$.

At the first glance, this result is easily explained: the second part of Proposition 1 says that the demand of each trader depends only on his private information and the equilibrium price. Thus the first and second terms in trading volume formula, which stands for trading volume induced by both informed and uninformed traders, do not change with the noisy per capita supply. The part of trading volume induced by liquidity trader is obviously an increasing function of the noisy per capita supply when $x_{1}>0$ and a decreasing function of the noisy per capita supply when $x_{1}<0$.

However, this conjecture is not correct. From the equilibrium price equation expressed in the first part of Proposition 1, for the equilibrium price given, the value of common information should change whenever the noisy per capita

\footnotetext{
${ }^{9}$ If we suppose that the noisy per capita supply is zero and that informed traders know the precision of uninformed traders' private informations' precisions $\left(\rho_{1}^{2}\right)$ but the latters do not the formers' precisions $\left(\rho_{1}^{1}\right)$, we get Blume, Easley \& O'Hara 1994's framework. It is not surprising that the results are the same.
} 
supply changes. More precisely, when the noisy per capita supply increases 1 , common information should increase $\frac{1}{u \rho_{1}^{s 1}+(1-u) \rho_{1}^{s^{2}}}$ to keep the equilibrium price unchanged. In other words, while the variances of the private information for both informed and uninformed trader retain, their probabilities change and so do the means of the private information. Recall that $V_{1}^{1} \mid \theta_{1}=u E\left[d_{1}\left(y_{1,1}^{j}\right) \mid \theta_{1}\right]$ where $y_{1,1}^{j} \mid \theta_{1} \sim N\left(\theta_{1}, 1 / \rho_{1}^{1}\right)$ and $V_{1}^{2} \mid=u E\left[d_{2}\left(y_{1,2}^{j}\right) \mid \theta_{1}\right]$ where $y_{1,2}^{j} \mid \theta_{1} \sim$ $N\left(\theta_{1}, 1 / \rho_{1}^{2}\right)$. The part of trading volume induced by both uninformed and informed traders change with the noisy per capita supply.

Whether trading volume induced by the uninformed and informed traders increases or decreases with the noisy per capita supply depends on the noisy per capita supply and other parameters of the market. However, the change of trading volume induced by liquidity traders is so strong that this effect always dominates and that the total trading volume always increases with the absolute value of the noisy per capita supply.

From Proposition 2, if traders look at only the equilibrium price, there are infinite solution of the noisy per capita supply and common information which verify the equilibrium price equation. If they look at both the equilibrium price and trading volume, there are only two possible solutions.

When $\rho_{t}^{w} \rightarrow \infty, \rho_{t}^{1}=\rho_{w}^{2}$, then $\rho_{t}^{s i} \rightarrow \rho_{t}^{1}=\rho_{t}^{2}, \theta_{t} \rightarrow \psi$, and we have the Brown \& Jenning 1989' framework. In their original paper, they suppose that traders look at only the equilibrium price and use the information contained in the equilibrium price. They show that the equilibrium is not revealing. The series of the equilibrium prices help traders to know the true value of the risky asset and the technical analysis is useful.

As Blume, Easley \& O'Hara 1994 argue, in the Brown \& Jenning 1989' framework if traders do know the equilibrium price and trading volume and use the information conveyed by the equilibrium price and trading volume, there is a revealing equilibrium: every trader will demand the same amount of the risky asset $d_{t}^{l}=d_{t}^{j}=d_{t}$ for all $l$ and $j$ and trading volume will be $\frac{1}{2}\left(\left|d_{t}\right|+\left|x_{t}-x_{t-1}\right|\right)$. In this setting, even though traders receive the private information with difference quality $\left(\rho_{t}^{1} \neq \rho_{t}^{2}\right)$, there exists always the same revealing equilibrium.

In our model, each trader makes his decision conditional on all information up to but not including the market statistics from their desired trades. The above calculation shows that without further assumption, traders can not infer the exact underlying supply uncertainty from trading volume and the equilibrium price is not revealing. The differential information that each trader owns 
does matter. Every trader makes his own decision according to his information and has his own different demand. Even though all traders receive their private information with the same quality as in Brown \& Jenning 1989' framework, their demands still differ.

\subsection{Equilibrium in the Second Period}

In this section, we investigate the equilibrium in the second period. To make the analysis tractable, we want the equilibrium in the first period to be revealing. The analysis in the last section show that if traders know the sign of the noisy per capita supply, they can infer the noisy per capita supply and common information from both the equilibrium price and trading volume. We assume that traders do know the sign of the noisy per capita supply although they do not know its value.

At the end of the first period, all traders have the same prior expectation again with mean $\bar{\theta}_{1}=\frac{\rho_{1}^{w} \theta_{1}+\rho_{0} \psi_{0}}{\rho_{0}+\rho_{1}^{w}}$ and variance $\left(\widehat{\rho}_{1}\right)^{-1}=\left(\rho_{0}+\rho_{1}^{w}\right)^{-1}$. Informed traders in group 1 then receive signals $y_{2}^{j}=\psi+w_{2}+e_{2}^{j}$ where the precision of the $e_{2}^{j}$ distribution is $\rho_{2}^{1}$. Similarly, uninformed traders in group 2 receive signals $y_{2}^{j}=\psi+w_{2}+\varepsilon_{2}^{j}$ where the precision of the $\varepsilon_{2}^{j}$ distribution is $\rho_{2}^{2}\left(\rho_{2}^{1}>\rho_{2}^{2}\right)$. Conditional his prior expectation and private information, each trader computes his expectation on the payoff of the risky asset, maximizes his expected wealth at date 3 and considers how to change his exposure of the risky asset. The following Proposition shows the equilibrium price and the demand of each trader in the second period.

Proposition 3 In the second period with proportional transaction costs,

(1) the equilibrium price is given by

$$
p_{2}=\frac{B \theta_{2}-C c+D-x_{2}}{A}
$$


where

$$
\begin{aligned}
A= & u\left(\widehat{\rho}_{1}+\rho_{2}^{s 1}\right)\left(1-\Phi\left(m_{1}\right)+\Phi\left(n_{1}\right)\right) \\
& +(1-u)\left(\widehat{\rho}_{1}+\rho_{2}^{s 2}\right)\left(1-\Phi\left(m_{2}\right)+\Phi\left(n_{2}\right)\right) \\
B= & u \rho_{2}^{s 1}\left(1-\Phi\left(m_{1}\right)+\Phi\left(n_{1}\right)\right)+(1-u) \rho_{2}^{s 2}\left(1-\Phi\left(m_{2}\right)+\Phi\left(n_{2}\right)\right) \\
C= & u\left(\widehat{\rho}_{1}+\rho_{2}^{s 1}\right)\left(1-\Phi\left(m_{1}\right)-\Phi\left(n_{1}\right)\right) \\
& +(1-u)\left(\widehat{\rho}_{1}+\rho_{2}^{s 2}\right)\left(1-\Phi\left(m_{2}\right)-\Phi\left(n_{2}\right)\right) \\
D= & \widehat{\rho}_{1} \bar{\theta}_{1}\left(1-u\left(\Phi\left(m_{1}\right)-\Phi\left(n_{1}\right)\right)-(1-u)\left(\Phi\left(m_{2}\right)-\Phi\left(n_{2}\right)\right)\right) \\
& +\left(u \rho_{1}^{s 1}\left(\Phi\left(m_{1}\right)-\Phi\left(n_{1}\right)\right)+(1-u) \rho_{1}^{s 2}\left(\Phi\left(m_{2}\right)-\Phi\left(n_{2}\right)\right)\right) \theta_{1} \\
& -u\left(\rho_{0}+\rho_{1}^{s 1}\right)\left(\Phi\left(m_{1}\right)-\Phi\left(n_{1}\right)\right) p_{1} \\
& -(1-u)\left(\rho_{0}+\rho_{1}^{s 2}\right)\left(\Phi\left(m_{2}\right)-\Phi\left(n_{2}\right)\right) p_{1} \\
& +\left(u\left(\Phi\left(m_{1}\right)-\Phi\left(n_{1}\right)\right)+(1-u)\left(\Phi\left(m_{2}\right)-\Phi\left(n_{2}\right)\right)\right) \rho_{0} \psi_{0} \\
& +\frac{u}{\sqrt{\chi_{2}^{1}}}\left(\phi\left(m_{1}\right)-\phi\left(n_{1}\right)\right)+\frac{1-u}{\sqrt{\chi_{2}^{2}}}\left(\phi\left(m_{2}\right)-\phi\left(n_{2}\right)\right) \\
m_{i}= & \sqrt{\chi_{2}^{i}}\left(\widehat{\rho}_{1}\left(p_{2}+c-\bar{\theta}_{1}\right)+\rho_{2}^{s i}\left(p_{2}+c\right)+\rho_{0}\left(\psi_{0}-p_{1}\right)-\rho_{1}^{s i} p_{1}-\chi_{1}^{i}\right) \\
n_{i}= & \sqrt{\chi_{2}^{i}}\left(\widehat{\rho}_{1}\left(p_{2}-c-\bar{\theta}_{1}\right)+\rho_{2}^{s i}\left(p_{2}-c\right)+\rho_{0}\left(\psi_{0}-p_{1}\right)-\rho_{1}^{s i} p_{1}-\chi_{1}^{i}\right) \\
\chi_{1}^{i}= & \rho_{2}^{s i} \theta_{2}-\rho_{1}^{s i} \theta_{1} \\
\chi_{2}^{i}= & \left(\frac{\left(\rho_{2}^{s i}\right)}{\rho_{2}^{i}}+\frac{\left(\rho_{1}^{s i}\right)^{2}}{\rho_{1}^{i}}\right)
\end{aligned}
$$

where $c$ is transaction cost per share, $\phi$ is the standard normal density, $\Phi$ is the standard normal cumulative distribution function, $\chi_{1}^{i}$ is the weighted change of the mean of the private information for traders in group $i$, and $\chi_{2}^{i}$ is the precision of the weighted change of the mean of the private information for traders in group $i$;

(2) the demand for the risky asset for each trader is given by

$d_{2}\left(y_{2, i}^{j}\right)=\left\{\begin{array}{l}\max \left(\widehat{\rho}_{1}\left(\bar{\theta}_{1}-p_{2}\right)+\rho_{2}^{s i}\left(y_{2, i}^{j}-p_{2}\right)+\left(\widehat{\rho}_{1}+\rho_{2}^{s i}\right) c, d_{1}\left(y_{1, i}^{j}\right)\right) \text { for buyer } \\ \min \left(\widehat{\rho}_{1}\left(\bar{\theta}_{1}-p_{2}\right)+\rho_{2}^{s i}\left(y_{2, i}^{j}-p_{2}\right)-\left(\widehat{\rho}_{1}+\rho_{2}^{s i}\right) c, d_{1}\left(y_{1, i}^{j}\right)\right) \text { for seller }\end{array}\right.$

The first part reflects investor's prior information, the second reflects the information surprise and the last reflects the effect of transaction costs.

When there are no transaction costs in the second period, $m_{i}=n_{i}, A=$ $u\left(\widehat{\rho}_{1}+\rho_{2}^{s 1}\right)+(1-u)\left(\widehat{\rho}_{1}+\rho_{2}^{s 2}\right), B=u \rho_{2}^{s 1}+(1-u) \rho_{2}^{s 2}$, and $D=\widehat{\rho}_{1} \bar{\theta}_{1}$. The 
equilibrium price simplifies to $\frac{\widehat{\rho}_{1} \bar{\theta}_{1}+\left(u \rho_{2}^{s 1}+(1-u) \rho_{2}^{s 2}\right) \theta_{2}-x_{2}}{u\left(\widehat{\rho}_{1}+\rho_{2}^{s 1}\right)+(1-u)\left(\widehat{\rho}_{1}+\rho_{2}^{s 2}\right)}$, and the demand of each trader simplifies to $\widehat{\rho}_{1}\left(\bar{\theta}_{1}-p_{2}\right)+\rho_{2}^{s i}\left(y_{2}^{j}-p_{2}\right)$, which are similar as those in the first period.

It is very interesting to compare the equilibrium price and the demand of each trader under proportional transaction costs with those under no transaction costs. As to the demand of each trader, the main difference is whether his demand in the second period depends on his demand in the first period. Under no transaction costs, the demand of each trader is totally determined by his private information, his prior (identical) expectation, and the equilibrium price. Because of the free transaction, it is almost always optimal to trade.

However, under proportional transaction costs, for each share bought or sold, a transaction cost $c$ has to be paid. If the gain from the change of his position is not high enough to compensate transaction costs, he prefers not to trade and his demand in the second period is that in the first period. If the gain is high enough to compensate the transaction costs, his demand changes and is expressed in the first term of the second part of Proposition 2. Thus his demand in the second period depends on that in the first period. The interpretation of each term in the demand of each trader is similar as that in Cheng 2005a's static model.

The difference of the demand of each trader between under no transaction costs and under proportional transaction costs leads to the difference of the equilibrium price between these two cases. When there are no transaction costs, the equilibrium price in the second period depends on all the parameters in the second period $\left(\rho_{2}^{s 1}, \rho_{2}^{s 2}, \theta_{2}, x_{2}\right)$ and only one part of the parameters in the first period: the initial identical expectation mean $\left(\psi_{0}\right)$ and precision $\left(\rho_{0}\right)$, the value of common information $\left(\theta_{1}\right)$ and its precision $\left(\rho^{w}\right)$, and the noisy per capita supply $\left(x_{1}\right)$. The precision of the private information are of no importance. Because these precision are necessary to know the equilibrium price in the first period, the equilibrium price in the second period does not depend on that in the first period.

When there are proportional transaction costs, because the demand of each trader in the second period depends on that in the first period and the latter depends all the parameters in the first period, the equilibrium price in the second period depends on all the parameters both in the second period and in the first period. The equilibrium price in the first period influences that in the second via $m_{i}$ and $n_{i}$. 
The transaction of each trader is the difference of his demand in the second period and that in the first period. From the second parts of Proposition 3 and of Proposition 1, it can be expressed as

$$
\begin{aligned}
& \triangle_{2, i}^{j}\left(y_{2, i}^{j}, y_{1, i}^{j}\right)=\triangle_{2, i}^{j}\left(Y_{i}^{j}\right) \\
= & \left\{\begin{array}{c}
\widehat{\rho}_{1}\left(\bar{\theta}_{1}-p_{2}\right)+\rho_{2}^{s i}\left(y_{2, i}^{j}-p_{2}\right)-\left(\widehat{\rho}_{1}+\rho_{2}^{s i}\right) c-d_{1, i}\left(y_{1}^{j}\right) \text { for buyer } \\
0 \quad \text { for others } \\
\widehat{\rho}_{1}\left(\bar{\theta}_{1}-p_{2}\right)+\rho_{2}^{s i}\left(y_{2, i}^{j}-p_{2}\right)+\left(\widehat{\rho}_{1}+\rho_{2}^{s i}\right) c-d_{1, i}\left(y_{1}^{j}\right) \text { for seller }
\end{array}\right. \\
= & \left\{\begin{array}{c}
\widehat{\rho}_{1}\left(\bar{\theta}_{1}-p_{2}\right)+Y_{i}^{j}-\rho_{2}^{s i} p_{2}+\rho_{1}^{s i} p_{1} \geq 0 \text { if } Y_{i}^{j}>\frac{m_{i}}{\sqrt{\chi_{2}^{i}}}+\chi_{1}^{i} \\
-\left(\widehat{\rho}_{1}+\rho_{2}^{s i}\right) c-\rho_{0}\left(\psi_{0}-p_{1}\right) \quad \text { if others } \\
0 \quad \\
\widehat{\rho}_{1}\left(\bar{\theta}_{1}-p_{2}\right)+Y_{i}^{j}-\rho_{2}^{s i} p_{2}+\rho_{1}^{s i} p_{1} \leq 0 \text { if } Y_{i}^{j}<\frac{n_{i}}{\sqrt{\chi_{2}^{i}}}+\chi_{1}^{i} \\
+\left(\widehat{\rho}_{1}+\rho_{2}^{s i}\right) c-\rho_{0}\left(\psi_{0}-p_{1}\right)
\end{array}\right.
\end{aligned}
$$

where $Y_{i}^{j}$ is the weighted change of trader $j$ 's private information $\left(Y_{i}^{j}=\rho_{2}^{s i} y_{2, i}^{j}\right.$ $\left.-\rho_{1}^{s i} y_{1, i}^{j}\right), \frac{m_{i}}{\sqrt{\chi_{2}^{i}}}+\chi_{1}^{i}$ are the up critical points of the weighted change of the private information above which traders in group $i$ will buy and $\frac{n_{i}}{\sqrt{\chi_{2}^{i}}}+\chi_{1}^{i}$ are the down critical points of the weighted change of the private information below which traders in group $i$ will sell. Trading volume in the second period is the weighted sum of the above transactions for both informed and informed traders plus the noisy per capita supply change.

Proposition 4 In the second period with proportional transaction costs, given $\theta_{2}$ and $x_{2}-x_{1}$, trading volume is

$$
V=\frac{u}{2} V_{2}^{1}+\frac{(1-u)}{2} V_{2}^{2}+\frac{\left|x_{2}-x_{1}\right|}{2}
$$

where

$$
V_{2}^{i}=\frac{1}{\sqrt{\chi_{2}^{i}}}\left(\phi\left(-m_{i}\right)-m_{i} \Phi\left(-m_{i}\right)+\phi\left(n_{i}\right)+n_{i} \Phi\left(n_{i}\right)\right)
$$

When there are no transaction costs, trading volume simplifies a little and $V_{2}^{i}$ becomes

$$
V_{2}^{i}=\frac{2}{\sqrt{\chi_{2}^{i}}} \phi\left(-\delta_{2}^{i} \sqrt{\chi_{2}^{i}}\right)-\delta_{2}^{i} \Phi\left(-\delta_{2}^{i} \sqrt{\chi_{2}^{i}}\right)+\delta_{2}^{i} \Phi\left(\delta_{2}^{i} \sqrt{\chi_{2}^{i}}\right)
$$


where

$$
\delta_{2}^{i}=\widehat{\rho}_{1}\left(p_{2}-\bar{\theta}_{1}\right)+\rho_{0}\left(\psi_{0}-p_{1}\right)+\rho_{2}^{s i}\left(p_{2}-\theta_{2}\right)-\rho_{1}^{s i}\left(p_{1}-\theta_{1}\right)
$$

It is worth noting that whether there are transaction costs or not, trading volume in the second periods always depends on all the parameters of the market in both periods. It is not surprising since each trader's transaction is always induced by the change of his private information together with the noisy per capita supply change and common information.

Proposition 5 In the second period with proportional transaction costs, (1) the equilibrium price is increasing in common information

$$
\frac{\partial p_{2}}{\partial \theta_{2}}=\frac{B}{A}>0
$$

(2) for the equilibrium price given, trading volume is a decreasing function of the noisy per capita supply change when $x_{2}-x_{1}<0$ and an increasing function of the noisy per capita supply change when $x_{2}-x_{1}>0$ and achieves its minimum at $x_{2}-x_{1}=0$.

Three remarks are in order. Firstly, although transaction costs change each trader's behavior and the equilibrium price, it does not change the economic logic between common information and the equilibrium price: the better common information is, the higher the traders' mean expectation on the payoff of the risky asset is, and the higher the equilibrium price is. Secondly, it is the noisy per capita supply change not the noisy per capita supply itself which matters to trading volume. Thirdly, comparison of Proposition 5 with Proposition 2 shows that transaction costs do not change the relation between trading volume, the noisy per capita supply change and the equilibrium price. Thus both informed and uninformed traders infer the value of common information and the noisy per capita supply change from the equilibrium price and trading volume in the second period.

\section{Trading Volume, Price Autocorrelation and Volatility with Transaction Costs}

As shown in the previous section, transaction costs influence each trader's behavior and the equilibrium price. Transaction costs influence the equilibrium 
price at date 2 in two ways: the dependence of the demand of each trader at date 2 on that at date 1 and the interval of no transaction with the length $2\left(\widehat{\rho}_{1}+\rho_{2}^{s i}\right) c$. The influence in the first way makes the equilibrium prices at date 1 and 2 correlated and the influence in the both ways changes return volatility. The influence of transaction costs on trading volume is investigated in the next subsection and the influences on price autocorrelation and return volatility are discussed in the second and third subsection, respectively.

\subsection{Transaction Costs and Trading Volume}

Proposition 6 In the second period with proportional transaction costs, trading volume $\left(V_{2}\right)$ is a decreasing convex function of transaction costs

$$
\begin{aligned}
\frac{\partial V_{2}}{\partial c}= & -\frac{u}{2}\left(\widehat{\rho}_{1}+\rho_{2}^{s 1}\right)\left(\Phi\left(-m_{1}\right)+\Phi\left(n_{1}\right)\right) \\
& -\frac{1-u}{2}\left(\widehat{\rho}_{1}+\rho_{2}^{s 2}\right)\left(\Phi\left(-m_{2}\right)+\Phi\left(n_{2}\right)\right) \\
< & 0 \\
\frac{\partial^{2} V_{2}}{\partial c^{2}}= & \frac{u}{2} \frac{\sqrt{\rho_{2}^{1}}}{\rho_{2}^{s 1}}\left(\widehat{\rho}_{1}+\rho_{2}^{s 1}\right)^{2}\left(\phi\left(m_{1}\right)+\phi\left(n_{1}\right)\right) \\
& +\frac{1-u}{2} \frac{\sqrt{\rho_{2}^{2}}}{\rho_{2}^{s 2}}\left(\widehat{\rho}_{1}+\rho_{2}^{s 2}\right)^{2}\left(\phi\left(m_{2}\right)+\phi\left(n_{2}\right)\right) \\
> & 0
\end{aligned}
$$

Trading volume decreases because traders do not trade at all if the weighted changes of their private signals that the traders receive in both periods are not significant enough $\left(\frac{n_{i}}{\sqrt{\chi_{2}^{i}}}+\chi_{1}^{i}<Y_{i}^{j}<\frac{m_{i}}{\sqrt{\chi_{2}^{i}}}+\chi_{1}^{i}\right)$. Even for those traders whose private signals' weighted changes are significant enough $\left(Y_{i}^{j}<\frac{n_{i}}{\sqrt{\chi_{2}^{i}}}+\chi_{1}^{i}\right.$ or $\left.Y_{i}^{j}>\frac{m_{i}}{\sqrt{\chi_{2}^{i}}}+\chi_{1}^{i}\right)$, they buy or sell less with the amount of $\left(\widehat{\rho}_{1}+\rho_{2}^{s i}\right) c$. Only traders who receive their private information $y_{1, i}^{j}=y_{1, i}^{*}=\frac{\rho_{0}\left(p_{1}-\psi_{0}\right)+\rho_{1}^{s i} p}{\rho_{1}^{s i}}$ in the first period and $y_{2, i}^{j}=y_{2, i}^{*}=\frac{\widehat{\rho}_{1}\left(p_{2}-\bar{\theta}_{1}\right)+\rho_{2}^{s i} p}{\rho_{2}^{s i}}$ in the second period at the same time do not change their demands which are, however, always zero regards of transaction costs.

The convexity of trading volume with respect to transaction costs means that when transaction costs are near to zero, the influence of transaction costs on trading volume is the most significant. On the contrary, when transaction costs are high, their marginal influence on trading volume is much smaller. 
When transaction costs are zero, $\frac{\partial V_{2}}{\partial c}$ achieves its minimum $-\frac{u}{2}\left(\widehat{\rho}_{1}+\rho_{2}^{s 1}\right)-$ $\frac{1-u}{2}\left(\widehat{\rho}_{1}+\rho_{2}^{s 2}\right)$.In the extreme case of infinite transaction costs, $\frac{\partial V_{2}}{\partial c}$ tends to 0 . Transaction costs are so high that it forbids any transaction. In fact, when transaction costs are infinite, $\frac{m_{i}}{\sqrt{\chi_{2}^{i}}}+\chi_{1}^{i}$ tends to positive infinite, $\frac{n_{i}}{\sqrt{\chi_{2}^{i}}}+\chi_{1}^{i}$ tends to negative infinite, and the intervals of no transaction $\frac{m_{i}-n_{i}}{\sqrt{\chi_{2}^{i}}}=2 c\left(\widehat{\rho}_{1}+\rho_{2}^{s i}\right)$ are also infinite. In this case, nobody transacts in the second period and every trader holds his position in the first period.

\subsection{Transaction Costs and Price Autocorrelation}

After Proposition 3 in the second section, we show that when there is no transaction costs, the equilibrium price in the second period does not depend on that in the first period and that when there is proportion transaction costs, the equilibrium price in the second does depend on that in the first period. In this subsection, we investigate the relation between transaction costs and price autocorrelation.

When there are no transaction costs, the autocorrelation between the equilibrium price at date 1 and that at date 2 is equal to

$$
\operatorname{corr}\left(\frac{\left(u \rho_{1}^{s 1}+(1-u) \rho_{1}^{s 2}\right) \theta_{1}-x_{1}}{\rho_{0}+u \rho_{1}^{s 1}+(1-u) \rho_{1}^{s 2}}, \frac{\left(u \rho_{2}^{s 1}+(1-u) \rho_{2}^{s 2}\right) \theta_{2}-x_{2}}{u\left(\widehat{\rho}_{1}+\rho_{2}^{s 1}\right)+(1-u)\left(\widehat{\rho}_{1}+\rho_{2}^{s 2}\right)}\right)
$$

Recall that $\theta_{1}=\psi+w_{1}, \theta_{2}=\psi+w_{2}$ and that common error $\left(w_{1}, w_{2}\right)$ and the noisy per capita supply $\left(x_{1}, x_{2}\right)$ are supposed to be independent, this autocorrelation is obviously zero.

When there are transaction costs, this correlation is no longer zero. Because we can not get the explicit solution to the equilibrium equation in the second period, it is impossible to express this autocorrelation explicitly. To understand the influence of proportional transaction costs on the autocorrelation, we calculate the partial derivative of $p_{2}$ with respect to $p_{1}$

$$
\frac{\partial p_{2}}{\partial p_{1}}=-\frac{u\left(\rho_{0}+\rho_{1}^{s 1}\right)\left(\Phi\left(m_{1}\right)-\Phi\left(n_{1}\right)\right)+(1-u)\left(\rho_{0}+\rho_{1}^{s 2}\right)\left(\Phi\left(m_{2}\right)-\Phi\left(n_{2}\right)\right)}{A}
$$

Since $m_{i} \geq n_{i}, \Phi\left(m_{i}\right) \geq \Phi\left(n_{i}\right)$. Together with $A>0$, the sign of this partial derivative is never positive. The equality holds when there are no transaction costs $\left(m_{i}=n_{i}\right)$, which is consistent with our analysis above. Otherwise, it is always negative. 
Proposition 7 In the large economy above,

(1) when there are no transaction costs, there is no price autocorrelation;

(2) when there are proportional transaction costs, there is price autocorrelation. For common information at date 2 given, the higher (lower) the equilibrium price at date 1, the lower (higher) the equilibrium price at date 2.

Recall that $\left[\frac{n_{i}}{\sqrt{\chi_{2}^{i}}}+\chi_{1}^{i}, \frac{m_{i}}{\sqrt{\chi_{2}^{i}}}+\chi_{1}^{i}\right]$ are the intervals of no transaction and $\frac{1}{2}\left[\frac{m_{i}+n_{i}}{\sqrt{\chi_{2}^{i}}}+\chi_{1}^{i}\right]$ are the mediums of the intervals for traders in group $i$. Our calculation show that these mediums are decreasing functions of the equilibrium price in the first period $\left(p_{1}\right)$

$$
\begin{aligned}
\frac{\partial\left(\frac{1}{2} \frac{m_{i}+n_{i}}{\sqrt{\chi_{2}^{i}}}+\chi_{1}^{i}\right)}{\partial p_{1}} & =\left(\widehat{\rho}_{1}+\rho_{2}^{s i}\right) \frac{\partial p_{2}}{\partial p_{1}}-\left(\rho_{0}+\rho_{1}^{s i}\right)-\frac{\partial \bar{\theta}_{1}}{\partial p_{1}} \widehat{\rho}_{1} \\
& =\left(\widehat{\rho}_{1}+\rho_{2}^{s i}\right) \frac{\partial p_{2}}{\partial p_{1}}-\left(\rho_{0}+\rho_{1}^{s i}\right)-\frac{\rho_{1}^{w}}{\left(\rho_{0}+\rho_{1}^{w}\right)^{2}} \frac{\partial \theta_{1}}{\partial p_{1}} \\
& <0
\end{aligned}
$$

It means that the higher the equilibrium price in the first period, the lower the mediums of the intervals of no transaction. Note that the third term is identical to both informed and uninformed traders. Since $\rho_{t}^{s 1}>\rho_{t}^{s 2}$, the influence of the equilibrium price at date 1 is more significant to informed traders than to uninformed traders. This move of the mediums makes the difference between total cancelled buy orders and total sell orders less (more) significant in case of positive (negative) common information and thus decreases the equilibrium price at date 2 .

To get an intuitional idea, let us look at a special case with $\theta_{1}=\psi_{0}$ and $x_{t}=0$. In this special case, $\theta_{1}=\psi_{0}$ and $x_{1}=0 \Rightarrow p_{1}=\theta_{1}=\psi_{0}$. Then $m_{i}$ and $n_{i}$ simplify to $\sqrt{\chi_{2}^{i}}\left(\widehat{\rho}_{1}\left(p_{2} \pm c-\bar{\theta}_{1}\right)+\rho_{2}^{s i}\left(p_{2} \pm c-\theta_{2}\right)\right)$. If $\theta_{2}=\bar{\theta}_{1}$, the mediums of the intervals simply to 0 . All cancelled buy orders are equal to all cancelled sell orders and the equilibrium price under proportional transaction costs is equal to that under no transaction $\operatorname{cots}\left(p_{2}=\theta_{2}=\bar{\theta}_{1}\right)$. If $\theta_{2}>\bar{\theta}_{1}$ $\left(\theta_{2}<\bar{\theta}_{1}\right)$, the mediums of the intervals are inferior (superior) to 0 . Then all canceled buy (sell) orders are superior to all cancelled sell (buy) orders and the equilibrium price when $\theta_{2}>\bar{\theta}_{1}\left(\theta_{2}<\bar{\theta}_{1}\right)$ is lower (higher) than that when $\theta_{2}=\bar{\theta}_{1}$. 


\subsection{Transaction Costs and Volatility}

Proportional transaction costs cancel both informed and uninformed traders' transaction. For each group of traders, not only buy orders but sell orders are reduced. If the total cancelled buy orders are always equal to the total cancelled sell orders regardless of proportional transaction costs, the equilibrium price should not change and volatility is independent on transaction costs. If not, they should be the functions of proportional transaction costs.

Proposition 8 In the second period with proportional transaction costs, the equilibrium price may be an increasing $(C<0)$ or decreasing $(C>0)$ function of proportional transaction costs.

From the equilibrium price equation expressed in Proposition 3, the partial partial derivative of the equilibrium price with respect to transaction costs can be calculated and is equal to $-\frac{C}{A}$ by Implicit Function Theorem. Since $A$ is always positive, the sigh of this partial derivative depends on that of $C$.

We know that $\frac{m_{i}}{\sqrt{\chi_{2}^{i}}}+\chi_{1}^{i}\left(\frac{n_{i}}{\sqrt{\chi_{2}^{i}}}+\chi_{1}^{i}\right)$ are the up (down) critical points of the change of the private information above (below) which traders in group $i$ will buy (sell) and $\frac{1}{2}\left[\frac{m_{i}+n_{i}}{\sqrt{\chi_{2}^{i}}}+\chi_{1}^{i}\right]$ are the mediums of these two critical points (interval of no transaction). While the mediums depend on parameters both at date 1 and at date 2 , the lengths of intervals $\left(2\left(\widehat{\rho}_{1}+\rho_{2}^{s i}\right) c\right)$ depend only on the parameters at date 2 .

The dependence of the mediums on the parameters at date 1 induces price autocorrelation, which has been studied in last subsection. Our calculation shows that the medium $\frac{1}{2}\left[\frac{m_{1}+n_{1}}{\sqrt{\chi_{2}^{1}}}+\chi_{1}^{1}\right]\left(\frac{1}{2}\left[\frac{m_{2}+n_{2}}{\sqrt{\chi_{2}^{2}}}+\chi_{1}^{2}\right]\right)$ is a decreasing (increasing) function of common information $\theta_{2}$. Because traders in group 1 (2) receive more (less) reliable information, it is economically logical that the higher common information, the lower (higher) the critical point of traders in group 1 (2) to buy.

Note that $\frac{C}{2}$ denote the cancelled buy orders minus the cancelled sell orders of all traders who still transact under proportional transaction costs (Refer to the proof of Proposition 3 in the Appendix). Because the cancelled sell orders and the cancelled buy orders are symmetric with respect to the mediums of no transaction intervals, the difference of the cancelled buy orders minus the cancelled sell orders of traders who do not transact under transaction costs have the same sign as $C$. Then the positive sign of $C$ means that transaction costs 
eliminates more buy orders than sell orders in total. Thus the equilibrium price under proportional transaction costs is lower than that under no transaction costs when $C$ is positive ${ }^{10}$.

In our model, volatility is measured by the variance of return ${ }^{11}$. Although the analytical result is not available because of the lack of closed-form solution of the equilibrium price at date 2, a special case helps us to understand the influence on return volatility.

\subsubsection{A Special Case with $\theta_{1}=\psi_{0}$ and $x_{t}=0$}

Proposition 9 In the special case with $\theta_{1}=\psi_{0}$ and $x_{1}=0$, conditional on the equilibrium price $p_{1}=\theta_{1}=\psi_{0}$ and prior identical expectation $\bar{\theta}_{1}$,

(1) the equilibrium price under proportional transaction costs and that under no transaction costs have the same mean : $E\left(p_{2}^{c} \mid \bar{\theta}_{1}, p_{1}=\theta_{1}\right)=E\left(p_{2}^{n} \mid \bar{\theta}_{1}, p_{1}=\theta_{1}\right)$ $=\bar{\theta}_{1}$, where $p_{2}^{c}$ and $p_{2}^{n}$ stand for the equilibrium prices under and under no transaction costs;

(2) return volatility under proportional transaction costs may be higher or lower than that under no transaction costs. More precisely,

$$
\begin{aligned}
& \operatorname{Var}\left(\frac{p_{2}^{c}-p_{1}}{p_{1}} \mid \bar{\theta}_{1}, p_{1}=\theta_{1}\right)>\operatorname{Var}\left(\frac{p_{2}^{n}-p_{1}}{p_{1}} \mid \bar{\theta}_{1}, p_{1}=\theta_{1}\right) \\
& \operatorname{Var}\left(\frac{p_{2}^{c}-p_{1}}{p_{1}} \mid \bar{\theta}_{1}, p_{1}=\theta_{1}\right)<\operatorname{Var}\left(\frac{p_{2}^{n}-p_{1}}{p_{1}} \mid \bar{\theta}_{1}, p_{1}=\theta_{1}\right)
\end{aligned} \quad \begin{aligned}
& \text { when } u>u^{*} \\
& \text { when }
\end{aligned}
$$

where

$$
u^{*}=\frac{1}{1-\frac{\left(\widehat{\rho}_{1}+\rho_{2}^{s 1}\right)\left(1-\Phi\left(m_{1}\right)-\Phi\left(n_{1}\right)\right)}{\left(\widehat{\rho}_{1}+\rho_{2}^{s 2}\right)\left(1-\Phi\left(m_{2}\right)-\Phi\left(n_{2}\right)\right)}}
$$

The conditional mean of the expected equilibrium price under proportional transaction costs does not change because the influence of transaction costs is symmetric when common information is positive or negative. Whether return volatility under proportional transaction costs is higher or lower than that under no transaction costs depends on whether the equilibrium price under proportional transaction costs is higher or lower than that under no transaction costs. The latter depends on the $\operatorname{sign}$ of $C$ as shown above. It is easy to show that

\footnotetext{
${ }^{10}$ In Cheng (2005)'s static model with a similar setting without the noisy supply shock, they have even stronger results: if $u$ is relatively small, the equilibrium price with proportional transaction costs is higher (lower) than that without transaction cost when common informaiton is positve (negative).

${ }^{11}$ Normally, price volatility is measured by the variance of the price and return volatility is measured by the variance of the price percent change. Since price volatility with defferent price means can not be directly comparable, we prefer return volatility in our model.
} 
when $u<u^{*}, C<0$ if $\theta_{2}>\bar{\theta}_{1}$, and $C>0$ if $\theta_{2}<\bar{\theta}_{1}{ }^{12}$.

\subsubsection{General Case}

Generally, we expect that return volatility is higher than that in the special case above for two reasons. First, the coefficient of the per capita noisy supply is bigger under proportional transaction costs than that under no transaction costs. By Implicit Function Theorem, we calculate the partial derivative of the equilibrium price with respect to the per capita supply $\frac{\partial p_{2}}{\partial x_{2}}=\frac{1}{A}$. Since $m_{i}>n_{i}$, $A<u\left(\widehat{\rho}_{1}+\rho_{2}^{s 1}\right)+(1-u)\left(\widehat{\rho}_{1}+\rho_{2}^{s 2}\right)$. It means that for the one unit change of the per capita supply, its influence on the equilibrium price under proportional transaction costs is more important than that under no transaction costs.

Second, there is price autocorrelation under proportional transaction costs. From Proposition 7, we know that for common information at date 2 given, the higher the equilibrium price at date 1 , the lower the equilibrium price at date 2 . This autocorrelation induces higher return volatility. For example, for $\theta_{2}=\bar{\theta}_{1}$, if $p_{1}=\psi_{0}$, then $p_{2}=\bar{\theta}_{1}$; if $p_{1}>\psi_{0}$, then $p_{2}<\bar{\theta}_{1}$; if $p_{1}<\psi_{0}$, then $p_{2}>\bar{\theta}_{1}$. Whenever $p_{1} \neq \psi_{0}$, the absolute value of the percent price change is higher than that when $p_{1}=\psi_{0}$.

\section{Related Empirical Research}

Some relevant empirical research includes Umlauf 1993, Jones \& Seguin 1997, Green, Maggioni \& Murinde 2000, and Hau 2006, among others. Sweden in the 1980s provides an excellent setting for a controlled laboratory-style experiment to determine how transaction costs (taxes) affect stock market behavior. Sweden stock market began without transaction taxes. In 1984 a 1\% round-trip tax was imposed on equity transactions and two years later the equity transaction tax rate was increased to $2 \%$. Umlauf 1993 studies the effects of transaction taxes on the behavior of Swedish equity returns and the main results are: (1) increasing transaction taxes resulted in a sharp drop in trading volume; (2) weekly to daily returns variance ratios declined during high-tax regimes, suggesting taxes induced greater negative autocorrelation in return; (3) all else being equal, taxes increase volatility.

\footnotetext{
${ }^{12}$ For more details about how proportional transaction costs influence the net cancelled orders of informed and uninformed traders in the very differenct way, please refer to Cheng 2005 a.
} 
Another similar example is in American financial market. On May 1, 1975, the lower and negotiated commissions on U.S. national stock exchange are introduced. Jones \& Seguin 1997 use the data of New York Stock Exchange (NYSE), the American Stock Exchange (AMEX) and National Association of Securities Dealers Automatic Quotations (NASDAQ) to investigate the relation between the volatility and this reduction in transaction $\operatorname{costs}^{13}$. Two main results of Jones \& Seguin 1997's empirical paper are: (1) the aggregate NYSE/AMEX portfolio exhibits reliably less volatility after the reduction in transaction costs; (2) the results are similar for all but the smallest size-based portfolio.

By using the data in the London Stock Exchange from 1870 to 1986, Green, Maggioni \& Murinde 2000 study the impact of transaction costs on market volatility and find that the sign of the relationship between transaction costs and market volatility is positive. This relationship in French stock between 1995 and 1999 is studied by Hau 2006. During this period, French stocks were subject to an important transaction cost increase whenever their price moved above the French franc (FF) 500 price threshold. Above FF 500, the minimal tick size for quotes increased by a factor of 10 from FF 0.1 to FF 1 , which constitutes an exogenous cost component induced by pricing grid of the electronic order book. He concludes that the effect of transaction costs on volatility is positive and significant, both statistically and economically.

Our model can explain these results quite well. The facts that transaction costs induce sharp drop of trading volume and (greater) negative autocorrelation is consistent with our analysis in subsections 3.1 and 3.2. From the second part of Proposition 9, we know that when $\rho_{2}^{1} \rightarrow \rho_{2}^{2}, \frac{\left(\widehat{\rho}_{1}+\rho_{2}^{s 1}\right)\left(1-\Phi\left(m_{1}\right)-\Phi\left(n_{1}\right)\right)}{\left(\widehat{\rho}_{1}+\rho_{2}^{s 2}\right)\left(1-\Phi\left(m_{2}\right)-\Phi\left(n_{2}\right)\right)} \rightarrow 1$, thus $u^{*} \rightarrow \infty$. It means that the condition in which return volatility under proportional transaction costs are higher than that under no transaction costs is always verified. For big-size (high stock price from FF 400 to FF 600) companies, so many market participants search the relevant informations and the differences of the precision between informed and uninformed traders are small. Consequently, their return volatility under transaction costs is higher than that under no transaction costs for these big-size companies, as shown in Jones \& Seguin 1997's empirical paper. In fact, Jones \& Seguin 1997 and Umlauf 1993's first result show that in general, the differences of precision between informed and uninformed traders are quite small that the whole market return volatility

\footnotetext{
${ }^{13}$ Regulated commissions are similar to transaction taxes since both are fixed in amount and levied on parties whenever a securities transaction occurs. Thus the event mentioned above is analogous to a one-time reduction in a tax on equity transactions.
} 
increase in transaction costs.

\section{Conclusions}

We develop a two periods model in which traders have differential information about the true value of the risky asset and trade the risky asset with proportional transaction costs. We assume that traders maximize their wealth in the end conditional on all information up to but not including the market statistics resulting from their desired trade. We show that without additional assumption, trading volume can not totally remove the noise in the pricing equation. However, because trading volume increases in the absolute value of noisy per capita supply change, it provides useful information on the asset fundamental value which cannot be inferred from the equilibrium price.

We then investigate the relation between trading volume, price autocorrelation, return volatility and proportional transaction costs. Trading volume decreases in proportional transaction costs and the influence of proportional transaction costs decreases at the margin. The result that price autocorrelation can be generated only by proportional transaction costs is interesting: under no transaction costs, the equilibrium prices at date 1 and 2 are not correlated (independent); however under proportional transaction costs, they are correlated the higher the equilibrium price at date 1 , the lower the equilibrium price at date 2. Contrary to "conventional wisdom" on the relation between return volatility and transaction costs, we show that return volatility may be and in general is increasing with proportional transaction costs, which is consistent with avaible empirical evidence. 


\section{Appendix}

Proof. of Proposition 1: At date 1, each trader maximizes by choice of $d_{1, i}^{j}$ the following function

$$
\begin{aligned}
& \max \left[E\left(-\exp \left(-w_{3, i}^{j}\right)\right)\right] \\
\Leftrightarrow & \max \left[\exp \left(E\left(w_{3, i}^{j} \mid \digamma_{1, i}^{j}\right)-\frac{1}{2} \operatorname{Var}\left(w_{3, i}^{j} \mid \digamma_{1, i}^{j}\right)\right)\right]
\end{aligned}
$$

where the wealth at date 3 is

$$
w_{3, i}^{j}=d_{1, i}^{j}\left(\psi-p_{1}\right)+z_{0}
$$

The first order condition for a maximum gives

$$
d_{1, i}^{j}=\frac{E\left(\psi \mid \digamma_{1, i}^{j}\right)-p_{1}}{V\left(\psi \mid \digamma_{1, i}^{j}\right)}
$$

By Bayes' Rule, the conditional expectation and conditional variance at date 1 are

$$
\begin{aligned}
E\left(\psi \mid \digamma_{1, i}^{j}\right) & =\frac{\rho_{0} \psi_{0}+\rho_{1}^{s i} y_{1, i}^{j}}{\rho_{0}+\rho_{1}^{s i}} \\
V\left(\psi \mid \digamma_{1, i}^{j}\right) & =\frac{1}{\rho_{0}+\rho_{1}^{s i}}
\end{aligned}
$$

Substituting the conditional expectation and conditional variance gives the demand at date 1 expressed in the second part of Proposition 1.

The total demand of the market is

$$
\begin{aligned}
\sum_{j=1}^{N} d_{1}\left(y_{1, i}^{j}\right)= & \sum_{j=1}^{u N} d_{1}\left(y_{1,1}^{j}\right)+\sum_{j=u N+1}^{N} d_{1}\left(y_{1,2}^{j}\right) \\
= & \sum_{j=1}^{u N}\left(\rho_{0}\left(\psi_{0}-p_{1}\right)+\rho_{1}^{s 1}\left(y_{1,1}^{j}-p_{1}\right)\right) \\
& +\sum_{j=u N+1}^{N}\left(\rho_{0}\left(\psi_{0}-p_{1}\right)+\rho_{1}^{s 2}\left(y_{1,2}^{j}-p_{1}\right)\right)
\end{aligned}
$$

By the Strong Law of Large Numbers as $u N$ and $(1-u) N \rightarrow \infty$, the total 
demand of the market can be rewritten as

$$
\begin{aligned}
\sum_{j=1}^{N} d_{1}\left(y_{1, i}^{j}\right)= & N \rho_{0} \psi_{0}-N\left(\rho_{0}+u \rho_{1}^{s 1}+(1-u) \rho_{1}^{s 2}\right) p_{1} \\
& +N\left(u \rho_{1}^{s 1}+(1-u) \rho_{1}^{s 2}\right) \theta_{1} \\
= & N x_{1}
\end{aligned}
$$

In the last equality, the fact that the total demand of the market should be equal to the noisy per capita supply in the equilibrium is used. After several arrangements, we have the equilibrium price expressed in the first part of Proposition 1.

Recall that in our model we define per capital volume as

$$
V=\frac{1}{2 N}\left(\sum_{j=1}^{u N}\left|d_{1}\left(y_{1,1}^{j}\right)\right|+\sum_{j=u N+1}^{N}\left|d_{1}\left(y_{1,2}^{j}\right)\right|\right)+\frac{\left|x_{1}\right|}{2}
$$

By the Strong Law of Large Numbers as $u N$ and $(1-u) N \rightarrow \infty$, this sequence of trading volume series converges almost surely to

$$
\frac{1}{2}\left(u E\left|d_{1}\left(y_{1,1}^{j}\right)\right|+(1-u) E\left|d_{1}\left(y_{1,2}^{j}\right)\right|+\left|x_{1}\right|\right)
$$

Using the lemma proved in Cheng 2005a with

$$
\begin{aligned}
x_{i}^{j} & =\alpha+\beta y_{i} \\
\alpha & =\rho_{0}\left(\psi_{0}-p_{1}\right)-\rho_{1}^{s i} p_{1} \\
\beta & =\rho_{1}^{s i} \\
y_{i} & \sim N\left(\theta_{1},\left(\rho_{1}^{i}\right)^{-1}\right)
\end{aligned}
$$

we get trading volume expressed in the third part of Proposition 1 immediately.

Proof. of Proposition 2: We first prove the case $x_{1}<0$. Differentiating trading volume expressed in the third part of Proposition 1 with respect to the noisy per capita supply gives

$$
\frac{\partial V_{1}}{\partial x_{1}}=\frac{1}{2}\left(\frac{\partial V_{1}^{1}}{\partial x_{1}}+\frac{\partial V_{1}^{2}}{\partial x_{1}}-1\right)
$$


where

$$
\begin{aligned}
\frac{\partial V_{1}^{i}}{\partial x_{1}}= & 2 \frac{\rho_{1}^{s i}}{\sqrt{\rho_{1}^{i}}} \phi\left(\frac{\delta_{1}^{i} \sqrt{\rho_{1}^{i}}}{\rho_{1}^{s i}}\right)\left(-\frac{\delta_{1}^{i} \sqrt{\rho_{1}^{i}}}{\rho_{1}^{s i}}\right) \frac{\sqrt{\rho_{1}^{i}}}{\rho_{1}^{s i}} \frac{\partial\left(\delta_{1}^{i}\right)}{\partial x_{1}} \\
& +\frac{\partial\left(\delta_{1}^{i}\right)}{\partial x_{1}}\left(\Phi\left(\frac{\delta_{1}^{i} \sqrt{\rho_{1}^{i}}}{\rho_{1}^{s i}}\right)-\Phi\left(-\frac{\delta_{1}^{i} \sqrt{\rho_{1}^{i}}}{\rho_{1}^{s i}}\right)\right) \\
& -\delta_{1}^{i} \phi\left(-\frac{\delta_{1}^{i} \sqrt{\rho_{1}^{i}}}{\rho_{1}^{s i}}\right)\left(\frac{-\sqrt{\rho_{1}^{i}}}{\rho_{1}^{s i}}\right) \frac{\partial\left(\delta_{1}^{i}\right)}{\partial x_{1}} \\
& +\delta_{1}^{i} \phi\left(\frac{\delta_{1}^{i} \sqrt{\rho_{1}^{i}}}{\rho_{1}^{s i}}\right)\left(\frac{\sqrt{\rho_{1}^{i}}}{\rho_{1}^{s i}}\right) \frac{\partial\left(\delta_{1}^{i}\right)}{\partial x_{1}} \\
= & \frac{\partial\left(\delta_{1}^{i}\right)}{\partial x_{1}}\left(\Phi\left(\frac{\delta_{1}^{i} \sqrt{\rho_{1}^{i}}}{\rho_{1}^{s i}}\right)-\Phi\left(-\frac{\delta_{1}^{i} \sqrt{\rho_{1}^{i}}}{\rho_{1}^{s i}}\right)\right)
\end{aligned}
$$

From the equilibrium price, we can express $\theta_{1}$ by $p_{1}$

$$
\theta_{1}=\frac{\left(\rho_{0}+u \rho_{1}^{1}+(1-u) \rho_{1}^{2}\right) p_{1}-\rho_{0} \psi_{0}+x_{1}}{u \rho_{1}^{1}+(1-u) \rho_{1}^{2}}
$$

Then $\delta_{1}^{i}$ can be written in another way

$$
\begin{aligned}
\delta_{1}^{i} & =\rho_{0}\left(\psi_{0}-p_{1}\right)+\rho_{1}^{s i}\left(\theta_{1}-p_{1}\right) \\
& =\rho_{0}\left(\psi_{0}-p_{1}\right)+\rho_{1}^{s i}\left(\frac{\left(\rho_{0}+u \rho_{1}^{s 1}+(1-u) \rho_{1}^{s 2}\right) p_{1}-\rho_{0} \psi_{0}+x_{1}}{\left(u \rho_{1}^{s 1}+(1-u) \rho_{1}^{s 2}\right)}-p_{1}\right) \\
& =\rho_{0}\left(\psi_{0}-p_{1}\right)+\rho_{1}^{s i}\left(\frac{\rho_{0}\left(p_{1}-\psi_{0}\right)+x_{1}}{\left(u \rho_{1}^{s 1}+(1-u) \rho_{1}^{s 2}\right)}\right) \\
& = \begin{cases}\frac{\rho_{0}\left(\psi_{0}-p_{1}\right)(1-u)\left(\rho_{1}^{s 2}-\rho_{1}^{s 1}\right)+\rho_{1}^{s 1} x}{u \rho_{1}^{s 1}+(1-u) \rho_{1}^{s 2}} \text { for } i=1 \\
\frac{\rho_{0}\left(\psi_{0}-p_{1}\right) u\left(\rho_{1}^{s 1}-\rho_{1}^{s 2}\right)+\rho_{1}^{s 2} x}{u \rho_{1}^{s 1}+(1-u) \rho_{1}^{s 2}} & \text { for } i=2\end{cases}
\end{aligned}
$$

Thus we have

$$
\begin{aligned}
\frac{\partial V_{1}}{\partial x_{1}}= & -\frac{1}{2}+\frac{u}{2} \frac{\rho_{1}^{s 1}}{u \rho_{1}^{s 1}+(1-u) \rho_{1}^{s 2}}\left(\Phi\left(\frac{\delta_{1}^{1} \sqrt{\rho_{1}^{1}}}{\rho_{1}^{s 1}}\right)-\Phi\left(-\frac{\delta_{1}^{1} \sqrt{\rho_{1}^{1}}}{\rho_{1}^{s 1}}\right)\right) \\
& +\frac{1-u}{2} \frac{\rho_{1}^{s 2}}{u \rho_{1}^{s 1}+(1-u) \rho_{1}^{s 2}}\left(\Phi\left(\frac{\delta_{1}^{2} \sqrt{\rho_{1}^{2}}}{\rho_{1}^{s 2}}\right)-\Phi\left(-\frac{\delta_{1}^{2} \sqrt{\rho_{1}^{2}}}{\rho_{1}^{s 2}}\right)\right)
\end{aligned}
$$




$$
\begin{aligned}
& <-\frac{1}{2}+\frac{u}{2} \frac{\rho_{1}^{s 1}}{u \rho_{1}^{s 1}+(1-u) \rho_{1}^{s 2}}+\frac{1-u}{2} \frac{\rho_{1}^{s 2}}{u \rho_{1}^{s 1}+(1-u) \rho_{1}^{s 2}} \\
& =-\frac{1}{2}+\frac{1}{2} \frac{u \rho_{1}^{s 1}+(1-u) \rho_{1}^{s 2}}{u \rho_{1}^{s 1}+(1-u) \rho_{1}^{s 2}} \\
& =0
\end{aligned}
$$

In the same way, we can prove that when $x_{1}>0, \frac{\partial V_{1}}{\partial x_{1}}>0$.

Proof. of Proposition 3: At date 2, each trader buys or sells according to the common prior and his private information at date 2 . The wealth constraint in this period is

$$
d_{2, i}^{j} p_{2}+z_{2, i}^{j}+\left|d_{2, i}^{j}-d_{1, i}^{j}\right| c=d_{1, i}^{j} p_{2}+z_{1, i}^{j}
$$

His wealth at date 3 is

$$
\begin{aligned}
w_{3}^{j}= & d_{2, i}^{j} \psi+z_{2, i}^{j} \\
= & d_{2, i}^{j} \psi+d_{1, i}^{j} p_{2}+z_{1, i}^{j}-d_{2, i}^{j} p_{2}-\left|d_{2, i}^{j}-d_{1, i}^{j}\right| c \\
= & d_{2, i}^{j} \psi+d_{1, i}^{j} p_{2}+z_{1, i}^{j}-d_{2, i}^{j} p_{2}-\left(d_{2, i}^{j}-d_{1, i}^{j}\right) c \text { for buyer at date } 2 \\
& d_{2, i}^{j} \psi+d_{1, i}^{j} p_{2}+z_{1, i}^{j}-d_{2, i}^{j} p_{2}+\left(d_{2, i}^{j}-d_{1, i}^{j}\right) c \text { for seller at date } 2
\end{aligned}
$$

Each trader maximizes his expected utility of wealth

$$
\begin{aligned}
\max & {\left[E\left(-\exp \left(-w_{3, i}^{j}\right)\right)\right] } \\
\Leftrightarrow & \max \left[\exp \left(E\left(w_{3, i}^{j} \mid \digamma_{2, i}^{j}\right)-\frac{1}{2} \operatorname{Var}\left(w_{3, i}^{j} \mid \digamma_{2, i}^{j}\right)\right)\right]
\end{aligned}
$$

As in the first periods, the first order condition for a maximum gives

$$
d_{2, i}^{j}=\left\{\begin{array}{l}
\frac{E\left(\psi \mid \digamma_{2, i}^{j}\right)-p_{2}-c}{V\left(\psi \mid \digamma_{2, i}^{j}\right)} \text { for buyer at date } 2 \\
\frac{E\left(\psi \mid \digamma_{2, i}^{j}\right)-p_{2}+c}{V\left(\psi \mid \digamma_{2, i}^{j}\right)} \text { for seller at date } 2
\end{array}\right.
$$

Recall that both informed and uninformed traders know all the information from the market in the first period and again have a common prior expectation on the risky asset's true value. By Bayes' Rule, the conditional expectation and 
conditional variance at date 2 are

$$
\begin{aligned}
E\left(\psi \mid \digamma_{2, i}^{j}\right) & =\frac{\widehat{\rho}_{1} \bar{\theta}_{1}+\rho_{2}^{s i} y_{2, i}^{j}}{\widehat{\rho}_{1}+\rho_{2}^{s i}} \\
V\left(\psi \mid \digamma_{2, i}^{j}\right) & =\frac{1}{\widehat{\rho}_{1}+\rho_{2}^{s i}}
\end{aligned}
$$

Substituting the conditional expectation and conditional variance gives

$$
d_{2, i}^{j}=\left\{\begin{array}{l}
\widehat{\rho}_{1}\left(\bar{\theta}_{1}-p_{2}\right)+\rho_{2}^{s i}\left(y_{2, i}^{j}-p_{2}\right)-\left(\widehat{\rho}_{1}+\rho_{2}^{s i}\right) \text { c for buyer at date } 2 \\
\widehat{\rho}_{1}\left(\bar{\theta}_{1}-p_{2}\right)+\rho_{2}^{s i}\left(y_{2, i}^{j}-p_{2}\right)+\left(\widehat{\rho}_{1}+\rho_{2}^{s i}\right) c \text { for seller at date } 2
\end{array}\right.
$$

Thus the demand for the risky asset $d_{2, i}\left(y_{2, i}^{j}, y_{1, i}^{j}\right)$ is the maximum of $d_{2, i}^{j}$ and $d_{1, i}^{j}$ for buyer in the second period and the minimum of $d_{2, i}^{j}$ and $d_{1, i}^{j}$ for seller in the second period.

To calculate the equilibrium price, we introduce a function

$$
f\left(y_{2, i}^{j}, y_{1, i}^{j}\right)=\left\{\begin{array}{l}
+1 \text { if } \rho_{2}^{s i} y_{2, i}^{j}-\rho_{1}^{s i} y_{1, i}^{j}>y_{i}^{u p} \\
\frac{\widehat{\rho}_{1}\left(\bar{\theta}_{1}-p_{2}\right)-\rho_{0}\left(\psi_{0}-p_{1}\right)+\rho_{2}^{s i} y_{2, i}^{j}-\rho_{1}^{s i} y_{1, i}^{j}-\rho_{2}^{s i} p_{2}+\rho_{1}^{s i} p_{1}}{c\left(\widehat{\rho}_{1}+\rho_{2}^{s 1}\right)} \text { if others } \\
-1 \text { if } \rho_{2}^{s i} y_{2, i}^{j}-\rho_{1}^{s i} y_{1, i}^{j}<y_{i}^{d o w n}
\end{array}\right.
$$

where

$$
\begin{aligned}
y_{i}^{\text {up }} & =\widehat{\rho}_{1}\left(p_{2}+c-\bar{\theta}_{1}\right)+\rho_{2}^{s i}\left(p_{2}+c\right)+\rho_{0}\left(\psi_{0}-p_{1}\right)-\rho_{1}^{s i} p_{1} \\
y_{i}^{\text {down }} & =\widehat{\rho}_{1}\left(p_{2}-c-\bar{\theta}_{1}\right)+\rho_{2}^{s i}\left(p_{2}-c\right)+\rho_{0}\left(\psi_{0}-p_{1}\right)-\rho_{1}^{s i} p_{1}
\end{aligned}
$$

and

$$
\begin{aligned}
\rho_{2}^{s i} y_{2, i}^{j}-\rho_{1}^{s i} y_{1, i}^{j} & \sim N\left(\chi_{1}^{i},\left(\chi_{2}^{i}\right)^{-1}\right) \\
& =N\left(\rho_{2}^{s i} \theta_{2}-\rho_{1}^{s i} \theta_{1}, \frac{\left(\rho_{2}^{s i}\right)^{2}}{\rho_{2}^{i}}+\frac{\left(\rho_{1}^{s i}\right)^{2}}{\rho_{1}^{i}}\right)
\end{aligned}
$$

Then the demand of each trader can be expressed in the following way

$$
d_{2, i}\left(y_{2, i}^{j}, y_{1, i}^{j}\right)=\left(\widehat{\rho}_{1} \bar{\theta}_{1}+\rho_{2}^{s i} y_{2, i}^{j}\right)-\left(\widehat{\rho}_{1}+\rho_{2}^{s i}\right)\left(p_{2}+f\left(y_{2, i}^{j}, y_{1, i}^{j}\right) c\right)
$$


The total demand of the market is

$$
\begin{aligned}
\sum_{j=1}^{N} d_{2}\left(y_{2, i}^{j}, y_{1, i}^{j}\right)= & \sum_{j=1}^{u N} d_{2,1}\left(y_{2,1}^{j}, y_{1,1}^{j}\right)+\sum_{j=u N+1}^{N} d_{2,2}\left(y_{2,2}^{j}, y_{1,2}^{j}\right) \\
= & \sum_{j=1}^{u N}\left(\widehat{\rho}_{1} \bar{\theta}_{1}+\rho_{2}^{s 1} y_{2,1}^{j}-\left(\widehat{\rho}_{1}+\rho_{2}^{s 1}\right)\left(p_{2}+f\left(y_{2,1}^{j}, y_{1,1}^{j}\right) c\right)\right) \\
& +\sum_{j=u N+1}^{N}\left(\widehat{\rho}_{1} \bar{\theta}_{1}+\rho_{2}^{s 2} y_{2,2}^{j}-\left(\widehat{\rho}_{1}+\rho_{2}^{s 2}\right)\left(p_{2}+f\left(y_{2,2}^{j}, y_{1,2}^{j}\right) c\right)\right)
\end{aligned}
$$

By the Strong Law of Large Numbers as $u N$ and $(1-u) N \rightarrow \infty$, the total demand of the market can be rewritten as

$$
\begin{aligned}
\sum_{j=1}^{N} d_{2}\left(y_{2, i}^{j}, y_{1, i}^{j}\right)= & N \widehat{\rho}_{1} \bar{\theta}_{1}-N\left(u\left(\widehat{\rho}_{1}+\rho_{2}^{s 1}\right)+(1-u)\left(\widehat{\rho}_{1}+\rho_{2}^{s 2}\right)\right) p_{2} \\
& +N\left(u \rho_{2}^{s 1}+(1-u) \rho_{2}^{s 2}\right) \theta_{2} \\
& -N u\left(\widehat{\rho}_{1}+\rho_{2}^{s 1}\right) E\left[f\left(y_{2,1}^{j}, y_{1,1}^{j}\right)\right] c \\
& -N(1-u)\left(\widehat{\rho}_{1}+\rho_{2}^{s 2}\right) E\left[f\left(y_{2,2}^{j}, y_{1,2}^{j}\right)\right] c
\end{aligned}
$$

By the market clearing condition in the second period, the equilibrium price converges almost surely to

$$
\begin{aligned}
p_{2}= & \frac{1}{\widehat{\rho}_{1}+u \rho_{2}^{s 1}+(1-u) \rho_{2}^{s 2}}\left[\widehat{\rho}_{1} \bar{\theta}_{1}+\left(u \rho_{2}^{s 1}+(1-u) \rho_{2}^{s 2}\right) \theta_{2}-x_{2}\right. \\
& \left.-\left(u\left(\widehat{\rho}_{1}+\rho_{2}^{s 1}\right) E\left[f\left(y_{2,1}^{j}, y_{1,1}^{j}\right)\right]+(1-u)\left(\widehat{\rho}_{1}+\rho_{2}^{s 2}\right) E\left[f\left(y_{2,2}^{j}, y_{1,2}^{j}\right)\right]\right) c\right]
\end{aligned}
$$

where

$$
\begin{aligned}
& E\left[f\left(y_{2, i}^{j}, y_{1, i}^{j}\right)\right] \\
= & \int_{-\infty}^{y_{i}^{\text {down }}}(-1) \sqrt{\chi_{2}^{i}} \phi\left(\sqrt{\chi_{2}^{i}}\left(x-\chi_{1}^{i}\right)\right) d x \\
& +\int_{y_{i}^{\text {up }}}^{+\infty}(+1) \sqrt{\chi_{2}^{i}} \phi\left(\sqrt{\chi_{2}^{i}}\left(x-\chi_{1}^{i}\right)\right) d x \\
& +\int_{y_{i}^{\text {down }}}^{y_{i}^{\text {up }}} \widehat{\rho}_{1}\left(\bar{\theta}_{1}-p_{2}\right)-\rho_{0}\left(\psi_{0}-p_{1}\right)-\rho_{2}^{s i} p_{2}+\rho_{1}^{s i} p_{1}+\chi_{1}^{i} \\
& \left.* \sqrt{\chi_{1}^{i}}+\rho_{2}^{s i}\right) \\
& \left.\sqrt{\chi_{2}^{i}}\left(x-\chi_{1}^{i}\right)\right) d x
\end{aligned}
$$




$$
\begin{aligned}
& +\int_{y_{i}^{\text {down }}}^{y_{i}^{u p}} \frac{\left(x-\chi_{1}^{i}\right)}{c\left(\widehat{\rho}_{1}+\rho_{2}^{s i}\right)} \sqrt{\chi_{2}^{i}} \phi\left(\sqrt{\chi_{2}^{i}}\left(x-\chi_{1}^{i}\right)\right) d x \\
= & -\Phi\left(\sqrt{\chi_{2}^{i}} \phi\left(\sqrt{\chi_{2}^{i}}\left(y_{i}^{\text {down }}-\chi_{1}^{i}\right)\right)\right)+1-\Phi\left(\sqrt{\chi_{2}^{i}} \phi\left(\sqrt{\chi_{2}^{i}}\left(y_{i}^{u p}-\chi_{1}^{i}\right)\right)\right) \\
& +\frac{1}{c\left(\widehat{\rho}_{1}+\rho_{2}^{s i}\right)}\left(\widehat{\rho}_{1} \bar{\theta}_{1}-\left(\widehat{\rho}_{1}+\rho_{2}^{s i}\right) p_{2}-\rho_{0} \psi_{0}+\left(\rho_{0}+\rho_{1}^{s i}\right) p_{1}+\chi_{1}^{i}\right) \\
& *\left(\Phi\left(\sqrt{\chi_{2}^{i}}\left(y_{i}^{u p}-\chi_{1}^{i}\right)\right)-\Phi\left(\sqrt{\chi_{2}^{i}}\left(y_{i}^{\text {down }}-\chi_{1}^{i}\right)\right)\right) \\
& -\frac{1}{c\left(\widehat{\rho}_{1}+\rho_{2}^{s i}\right) \sqrt{\chi_{2}^{i}}}\left(\phi\left(\sqrt{\chi_{2}^{i}}\left(y_{i}^{u p}-\chi_{1}^{i}\right)\right)-\phi\left(\sqrt{\chi_{2}^{i}}\left(y_{i}^{\text {down }}-\chi_{1}^{i}\right)\right)\right)
\end{aligned}
$$

Substituting these two expectation gives the coefficients $A, B, C$, and $D$ and the equilibrium price follows after several arrangements.

Proof. of Proposition 4: From the second parts of Proposition 3 and of Proposition 1, the transaction of each trader in the second period is

$$
\begin{aligned}
& \triangle_{2, i}^{j}\left(y_{2, i}^{j}, y_{1, i}^{j}\right) \\
= & \left\{\begin{array}{c}
\widehat{\rho}_{1}\left(\bar{\theta}_{1}-p_{2}\right)+\rho_{2}^{s i}\left(y_{2, i}^{j}-p_{2}\right)-\left(\widehat{\rho}_{1}+\rho_{2}^{s i}\right) c-d_{1}\left(y_{1, i}^{j}\right) \text { for buyer } \\
0 \quad \text { for others } \\
\widehat{\rho}_{1}\left(\bar{\theta}_{1}-p_{2}\right)+\rho_{2}^{s i}\left(y_{2, i}^{j}-p_{2}\right)+\left(\widehat{\rho}_{1}+\rho_{2}^{s i}\right) c-d_{1}\left(y_{1, i}^{j}\right) \text { for seller }
\end{array}\right. \\
= & \left\{\begin{array}{c}
\widehat{\rho}_{1}\left(\bar{\theta}_{1}-p_{2}\right)+Y_{i}^{j}-\rho_{2}^{s i} p_{2}+\rho_{1}^{s i} p_{1} \\
-\left(\widehat{\rho}_{1}+\rho_{2}^{s i}\right) c-\rho_{0}\left(\psi_{0}-p_{1}\right) \\
0 \text { if others } Y_{i}^{j}>y_{i}^{u p} \\
\widehat{\rho}_{1}\left(\bar{\theta}_{1}-p_{2}\right)+Y_{i}^{j}-\rho_{2}^{s i} p_{2}+\rho_{1}^{s i} p_{1} \\
+\left(\widehat{\rho}_{1}+\rho_{2}^{s i}\right) c-\rho_{0}\left(\psi_{0}-p_{1}\right)
\end{array}\right.
\end{aligned}
$$

where

$$
Y_{i}^{j}=\rho_{2}^{s i} y_{2, i}^{j}-\rho_{1}^{s i} y_{1, i}^{j}
$$

Then trading volume is

$$
\begin{aligned}
V_{2} & =\frac{1}{2 N}\left(\sum_{j=1}^{u N} \triangle_{2,1}^{j}\left(Y_{1}^{j}\right)+\sum_{j=1+u N}^{N} \triangle_{2,2}^{j}\left(Y_{2}^{j}\right)\right)+\frac{\left|x_{2}-x_{1}\right|}{2} \\
& =\frac{1}{2}\left(u E\left(\triangle_{2,1}^{j}\left(Y_{1}^{j}\right) \mid \digamma_{2,1}^{j}\right)+(1-u) E\left(\triangle_{2,2}^{j}\left(Y_{2}^{j}\right) \mid \digamma_{2,2}^{j}\right)\right)+\frac{\left|x_{2}-x_{1}\right|}{2} \\
& =\frac{1}{2}\left(u V_{2}^{1}+(1-u) V_{2}^{2}\right)+\frac{\left|x_{2}-x_{1}\right|}{2}
\end{aligned}
$$


where

$$
V_{2}^{i}=\int_{-\infty}^{y_{i}^{\text {down }}}\left|\triangle_{2, i}^{j}\left(x_{i}^{j}\right)\right| f_{i}\left(x_{i}^{j}\right) d x_{i}^{j}+\int_{y_{i}^{u p}}^{\infty}\left|\triangle_{2, i}^{j}\left(x_{i}^{j}\right)\right| f_{i}\left(x_{i}^{j}\right) d x_{i}^{j}
$$

Using the lemma proved in Cheng 2005a with

$$
\begin{aligned}
x_{i}^{j} & =\alpha+\beta y_{i} \\
\alpha & =\widehat{\rho}_{1}\left(\bar{\theta}_{1}-p_{2}\right)-\rho_{2}^{s i} p_{2} \pm\left(\widehat{\rho}_{1}+\rho_{2}^{s i}\right) c-\rho_{0}\left(\psi_{0}-p_{1}\right)+\rho_{1}^{s i} p_{1} \\
\beta & =1 \\
y_{i} & \sim N\left(\chi_{1}^{i},\left(\chi_{2}^{i}\right)^{-1}\right)
\end{aligned}
$$

we have trading volume expressed in Proposition 4.

Proof. of Proposition 5: Because we could not get the explicit solution for the equilibrium price, we need to calculate first $\frac{\partial F}{\partial \theta_{2}}$ and $\frac{\partial F}{\partial p_{2}}$ where $F=$ $p_{2} A-B \theta_{2}+C c-D+x_{2}=0$.

$$
\frac{\partial F}{\partial \theta_{2}}=p_{2} \frac{\partial A}{\partial \theta_{2}}-\frac{\partial B}{\partial \theta_{2}} \theta_{2}+\frac{\partial C}{\partial \theta_{2}} c-\frac{\partial D}{\partial \theta_{2}}-B
$$

where

$$
\begin{aligned}
+\frac{\partial A}{\partial \theta_{2}} p_{2}= & -u\left(\widehat{\rho}_{1}+\rho_{2}^{s 1}\right)\left(\phi\left(m_{1}\right) \frac{\partial m_{1}}{\partial \theta_{2}}-\phi\left(n_{1}\right) \frac{\partial n_{1}}{\partial \theta_{2}}\right) p_{2} \\
& -(1-u)\left(\widehat{\rho}_{1}+\rho_{2}^{s 2}\right)\left(\phi\left(m_{2}\right) \frac{\partial m_{2}}{\partial \theta_{2}}-\phi\left(n_{2}\right) \frac{\partial n_{2}}{\partial \theta_{2}}\right) p_{2} \\
-\frac{\partial B}{\partial \theta_{2}} \theta_{2}= & u \rho_{2}^{s 1}\left(\phi\left(m_{1}\right) \frac{\partial m_{1}}{\partial \theta_{2}}-\phi\left(n_{1}\right) \frac{\partial n_{1}}{\partial \theta_{2}}\right) \theta_{2} \\
& +(1-u) \rho_{2}^{s 2}\left(\phi\left(m_{2}\right) \frac{\partial m_{2}}{\partial \theta_{2}}-\phi\left(n_{2}\right) \frac{\partial n_{2}}{\partial \theta_{2}}\right) \theta_{2} \\
+\frac{\partial C}{\partial \theta_{2}} c= & -u\left(\widehat{\rho}_{1}+\rho_{2}^{s 1}\right)\left(\phi\left(m_{1}\right) \frac{\partial m_{1}}{\partial \theta_{2}}+\phi\left(n_{1}\right) \frac{\partial n_{1}}{\partial \theta_{2}}\right) \\
& -(1-u)\left(\widehat{\rho}_{1}+\rho_{2}^{s 2}\right)\left(\phi\left(m_{2}\right) \frac{\partial m_{2}}{\partial \theta_{2}}+\phi\left(n_{2}\right) \frac{\partial n_{2}}{\partial \theta_{2}}\right) \\
-\frac{\partial D}{\partial \theta_{2}}= & u \widehat{\rho}_{1} \bar{\theta}_{1}\left(\phi\left(m_{1}\right) \frac{\partial m_{1}}{\partial \theta_{2}}-\phi\left(n_{1}\right) \frac{\partial n_{1}}{\partial \theta_{2}}\right) \\
& +(1-u) \widehat{\rho}_{1} \bar{\theta}_{1}\left(\phi\left(m_{2}\right) \frac{\partial m_{2}}{\partial \theta_{2}}-\phi\left(n_{2}\right) \frac{\partial n_{2}}{\partial \theta_{2}}\right)
\end{aligned}
$$




$$
\begin{aligned}
& -u \rho_{1}^{s 1}\left(\phi\left(m_{1}\right) \frac{\partial m_{1}}{\partial \theta_{2}}-\phi\left(n_{1}\right) \frac{\partial n_{1}}{\partial \theta_{2}}\right) \theta_{1} \\
& -(1-u) \rho_{1}^{s 2}\left(\phi\left(m_{2}\right) \frac{\partial m_{2}}{\partial \theta_{2}}-\phi\left(n_{2}\right) \frac{\partial n_{2}}{\partial \theta_{2}}\right) \theta_{1} \\
& +u\left(\rho_{0}+\rho_{1}^{s 1}\right)\left(\phi\left(m_{1}\right) \frac{\partial m_{1}}{\partial \theta_{2}}-\phi\left(n_{1}\right) \frac{\partial n_{1}}{\partial \theta_{2}}\right) p_{1} \\
& +(1-u)\left(\rho_{0}+\rho_{1}^{s 2}\right)\left(\phi\left(m_{2}\right) \frac{\partial m_{2}}{\partial \theta_{2}}-\phi\left(n_{2}\right) \frac{\partial n_{2}}{\partial \theta_{2}}\right) p_{1} \\
& -u\left(\phi\left(m_{1}\right) \frac{\partial m_{1}}{\partial \theta_{2}}-\phi\left(n_{1}\right) \frac{\partial n_{1}}{\partial \theta_{2}}\right) \rho_{0} \psi_{0} \\
& -(1-u)\left(\phi\left(m_{2}\right) \frac{\partial m_{2}}{\partial \theta_{2}}-\phi\left(n_{2}\right) \frac{\partial n_{2}}{\partial \theta_{2}}\right) \rho_{0} \psi_{0} \\
& -u \frac{1}{\sqrt{\chi_{2}^{i}}}\left(\phi\left(m_{1}\right)\left(-m_{1}\right) \frac{\partial m_{1}}{\partial \theta_{2}}-\phi\left(n_{1}\right)\left(-n_{1}\right) \frac{\partial n_{1}}{\partial \theta_{2}}\right) \\
& -(1-u) \frac{1}{\sqrt{\chi_{2}^{i}}}\left(\phi\left(m_{2}\right)\left(-m_{2}\right) \frac{\partial m_{2}}{\partial \theta_{2}}-\phi\left(n_{2}\right)\left(-n_{2}\right) \frac{\partial n_{2}}{\partial \theta_{2}}\right)
\end{aligned}
$$

After serval substitutions and arrangements, we have

$$
\frac{\partial F}{\partial \theta_{2}}=-B
$$

In the same way, we have

$$
\frac{\partial F}{\partial p_{2}}=A
$$

The partial derivative of the equilibrium price at date 2 with respect to common information follows directly from the use of Implicit Function Theorem. It is obvious that $A>0, B>0$.

For the second part of Proposition, we first prove the case $x_{2}-x_{1}<0$ as in the proof of Proposition 2. Differentiating trading volume expressed in Proposition 4 with respect to the noisy per capita supply change between date 1 and date 2

$$
\frac{\partial V_{2}}{\partial\left(x_{2}-x_{1}\right)}=\frac{1}{2}\left(\frac{\partial V_{2}^{1}}{\partial\left(x_{2}-x_{1}\right)}+\frac{\partial V_{2}^{2}}{\partial\left(x_{2}-x_{1}\right)}-1\right)
$$


where

$$
\begin{aligned}
\frac{\partial V_{2}^{i}}{\partial\left(x_{2}-x_{1}\right)}= & \frac{1}{\sqrt{\chi_{2}^{i}}} \phi\left(m_{i}\right)\left(-m_{i}\right) \frac{\partial\left(m_{i}\right)}{\partial\left(x_{2}-x_{1}\right)} \\
& -\frac{1}{\sqrt{\chi_{2}^{i}}} \frac{\partial\left(m_{i}\right)}{\partial\left(x_{2}-x_{1}\right)}\left(\Phi\left(-m_{i}\right)-m_{i} \phi\left(-m_{i}\right)\right) \\
& +\frac{1}{\sqrt{\chi_{2}^{i}}} \phi\left(n_{i}\right)\left(-n_{i}\right) \frac{\partial\left(n_{i}\right)}{\partial\left(x_{2}-x_{1}\right)} \\
& +\frac{1}{\sqrt{\chi_{2}^{i}}} \frac{\partial\left(n_{i}\right)}{\partial\left(x_{2}-x_{1}\right)}\left(\Phi\left(n_{i}\right)+n_{i} \phi\left(n_{i}\right)\right) \\
= & \frac{1}{\sqrt{\chi_{2}^{i}}} \frac{\partial\left(m_{i}\right)}{\partial\left(x_{2}-x_{1}\right)}\left(\Phi\left(n_{i}\right)-\Phi\left(-m_{i}\right)\right)
\end{aligned}
$$

Because $m_{i}$ contains $\theta_{2}$ and $\theta_{2}$ is a function of $\left(x_{2}-x_{1}\right)$ for the equilibrium price given, we need to calculate $\frac{\partial \theta_{2}}{\partial\left(x_{2}-x_{1}\right)}$ which is equal to $-\frac{\partial F}{\partial\left(x_{2}-x_{1}\right)} / \frac{\partial F}{\partial \theta_{2}}$ by Implicit Function Theorem. It is clear that $\frac{\partial F}{\partial\left(x_{2}-x_{1}\right)}=1$ and then $\frac{\partial \theta_{2}}{\partial\left(x_{2}-x_{1}\right)}=$ $\frac{1}{B}, \frac{\partial m_{i}}{\partial\left(x_{2}-x_{1}\right)}=\frac{\sqrt{\chi_{2}^{i}} \rho_{2}^{s i}}{B}$. Thus we have

$$
\begin{aligned}
\frac{\partial V_{2}}{\partial\left(x_{2}-x_{1}\right)}= & -\frac{1}{2}+\frac{u}{2} \frac{\rho_{2}^{s 1}}{B}\left(\Phi\left(n_{1}\right)-\Phi\left(-m_{1}\right)\right) \\
& +\frac{1-u}{2} \frac{\rho_{2}^{s 2}}{B}\left(\Phi\left(n_{1}\right)-\Phi\left(-m_{1}\right)\right) \\
< & -\frac{1}{2}+\frac{u}{2} \frac{\rho_{2}^{s 1}\left(1-\Phi\left(m_{1}\right)+\Phi\left(n_{1}\right)\right)}{B} \\
& +\frac{1-u}{2} \frac{\rho_{2}^{s 2}\left(1-\Phi\left(m_{2}\right)+\Phi\left(n_{2}\right)\right)}{B} \\
= & -\frac{1}{2}+\frac{1}{2} \frac{B}{B} \\
= & 0
\end{aligned}
$$

In the same way, we can prove that when $x_{2}-x_{1}>0, \frac{\partial V_{2}}{\partial\left(x_{2}-x_{1}\right)}>0$.

Proof. of Proposition 6: Differentiating trading volume expressed in the proportion 4 in the text with respect to $c$ yields

$$
\begin{aligned}
\frac{\partial V_{2}^{i}}{\partial c}= & \frac{1}{\sqrt{\chi_{2}^{i}}} \phi\left(m_{i}\right)\left(-m_{i}\right) \frac{\partial\left(m_{i}\right)}{\partial c} \\
& -\frac{1}{\sqrt{\chi_{2}^{i}}} \frac{\partial\left(m_{i}\right)}{\partial c}\left(\Phi\left(-m_{i}\right)-m_{i} \phi\left(-m_{i}\right)\right) \\
& +\frac{1}{\sqrt{\chi_{2}^{i}}} \phi\left(n_{i}\right)\left(-n_{i}\right) \frac{\partial\left(n_{i}\right)}{\partial c}
\end{aligned}
$$




$$
\begin{aligned}
& +\frac{1}{\sqrt{\chi_{2}^{i}}} \frac{\partial\left(n_{i}\right)}{\partial c}\left(\Phi\left(n_{i}\right)+n_{i} \phi\left(n_{i}\right)\right) \\
= & \frac{1}{\sqrt{\chi_{2}^{i}}}\left(\frac{\partial\left(n_{i}\right)}{\partial c} \Phi\left(n_{i}\right)-\frac{\partial\left(m_{i}\right)}{\partial c} \Phi\left(-m_{i}\right)\right) \\
= & -\left(\widehat{\rho}_{1}+\rho_{2}^{s i}\right)\left(\Phi\left(n_{i}\right)-\Phi\left(-m_{i}\right)\right) \\
< & 0 \\
\frac{\partial^{2} V_{2}^{i}}{\partial c^{2}}= & -\left(\widehat{\rho}_{1}+\rho_{2}^{s i}\right)\left(\phi\left(-m_{i}\right) \frac{\partial\left(-m_{i}\right)}{\partial c}+\phi\left(n_{i}\right) \frac{\partial\left(n_{i}\right)}{\partial c}\right) \\
= & \sqrt{\chi_{2}^{i}}\left(\widehat{\rho}_{1}+\rho_{2}^{s i}\right)^{2}\left(\phi\left(-m_{i}\right)+\phi\left(n_{i}\right)\right) \\
> & 0
\end{aligned}
$$

Thus $V_{2}$ is a decreasing convex function of $c$.

Proof. of Proposition 7: Where there are no transaction costs,

$$
\begin{aligned}
& \operatorname{corr}\left(p_{1}, p_{2}\right) \\
= & \operatorname{corr}\left(\frac{\left(u \rho_{1}^{s 1}+(1-u) \rho_{1}^{s 2}\right) \theta_{1}-x_{1}}{\rho_{0}+u \rho_{1}^{s 1}+(1-u) \rho_{1}^{s 2}}, \frac{\left(u \rho_{2}^{s 1}+(1-u) \rho_{2}^{s 2}\right) \theta_{2}-x_{2}}{u\left(\widehat{\rho}_{1}+\rho_{2}^{s 1}\right)+(1-u)\left(\widehat{\rho}_{1}+\rho_{2}^{s 2}\right)}\right) \\
= & \operatorname{corr}\left(\frac{\left(u \rho_{1}^{s 1}+(1-u) \rho_{1}^{s 2}\right) w_{1}-x_{1}}{\rho_{0}+u \rho_{1}^{s 1}+(1-u) \rho_{1}^{s 2}}, \frac{\left(u \rho_{2}^{s 1}+(1-u) \rho_{2}^{s 2}\right) w_{2}-x_{2}}{u\left(\widehat{\rho}_{1}+\rho_{2}^{s 1}\right)+(1-u)\left(\widehat{\rho}_{1}+\rho_{2}^{s 2}\right)}\right) \\
= & 0
\end{aligned}
$$

In the last equality, the fact that $w_{t}, x_{t}$ are independent is used.

When there are proportional transaction costs, we calculate the partial derivative of $p_{2}$ with respect to $p_{1}$. By Implicit Function Theorem,

$$
\frac{\partial p_{2}}{\partial p_{1}}=-\frac{\frac{\partial F}{\partial p_{1}}}{\frac{\partial F}{\partial p_{2}}}
$$

where $\frac{\partial F}{\partial p_{2}}=A$, and

$$
\frac{\partial F}{\partial p_{1}}=u\left(\rho_{0}+\rho_{1}^{s 1}\right)\left(\Phi\left(m_{1}\right)-\Phi\left(n_{1}\right)\right)+(1-u)\left(\rho_{0}+\rho_{1}^{s 2}\right)\left(\Phi\left(m_{2}\right)-\Phi\left(n_{2}\right)\right)
$$

Since $m_{i} \geq n_{i}$, thus $\Phi\left(m_{i}\right) \geq \Phi\left(n_{i}\right)$ and $\frac{\partial F}{\partial p_{1}}>0$. Together with $A>0$, the sign of $\frac{\partial p_{2}}{\partial p_{1}}$ is never positive. The equality holds when there are no transaction $\operatorname{costs}\left(m_{i}=n_{i}\right)$. Otherwise, it is always negative.

Proof. of Proposition 8: By Implicit Function Theorem, as in the proof of Proposition 5, we calculate the partial derivative of $p_{2}$ with respect to $c$ : 
$\frac{\partial p_{2}}{\partial c}=-\frac{\frac{\partial F}{\partial C}}{\partial F}=-\frac{C}{A}$. Since $A$ is always positive, the sigh of this partial derivative depends on that of $C$.

Proof. of Proposition 9: It is easy to check that when $\theta_{2}=\bar{\theta}_{1}$, whether there are transaction costs or not, the equilibrium price $p_{2}$ is always $\bar{\theta}_{1}$. To prove the first part of Proposition, it is enough to prove that $p_{2}$ is always symmetric with respect to $\bar{\theta}_{1}$. When there are no transaction costs, it is evident that this is true. When there are proportional transaction costs, we prove it by showing that

$$
p_{2}^{\bar{\theta}_{1}+\epsilon}+p_{2}^{\bar{\theta}_{1}-\epsilon}=2 \bar{\theta}_{1}
$$

where $p_{2}^{\bar{\theta}_{1}+\epsilon}$ and $p_{2}^{\bar{\theta}_{1}-\epsilon}$ are the correspondent equilibrium prices when common error is $+\epsilon$ or $-\epsilon$.

We write $p_{2}^{\bar{\theta}_{1}+\epsilon}$ in another way $\bar{\theta}_{1}+\zeta$. If we can show that $p_{2}^{\bar{\theta}_{1}-\epsilon}=\bar{\theta}_{1}-$ $\zeta$, the first part is proved. From the equilibrium price equation expressed in Proposition 3, we have

$$
\bar{\theta}_{1}+\zeta=\frac{B \theta_{2}-C c+D}{A}
$$

where A, B, C, D are expressed in Proposition 3, and

$$
\begin{aligned}
m_{i} & =\sqrt{\chi_{2}^{i}}\left(\widehat{\rho}_{1}\left(\bar{\theta}_{1}+\zeta+c-\bar{\theta}_{1}\right)+\rho_{2}^{s i}\left(\bar{\theta}_{1}+\zeta+c-\bar{\theta}_{1}-\epsilon\right)\right) \\
& =\sqrt{\chi_{2}^{i}}\left(\widehat{\rho}_{1}(\zeta+c)+\rho_{2}^{s i}(\zeta+c-\epsilon)\right) \\
n_{i} & =\sqrt{\chi_{2}^{i}}\left(\widehat{\rho}_{1}\left(\bar{\theta}_{1}+\zeta-c-\bar{\theta}_{1}\right)+\rho_{2}^{s i}\left(\bar{\theta}_{1}+\zeta-c-\bar{\theta}_{1}-\epsilon\right)\right) \\
& =\sqrt{\chi_{2}^{i}}\left(\widehat{\rho}_{1}(\zeta-c)+\rho_{2}^{s i}(\zeta-c-\epsilon)\right)
\end{aligned}
$$

Then $\zeta$ can be written as

$$
\begin{aligned}
\zeta & =p_{2}^{\bar{\theta}_{1}+\epsilon}-\bar{\theta}_{1}=p_{2}^{\bar{\theta}_{1}+\epsilon}-\frac{A}{A} \bar{\theta}_{1} \\
& =\frac{-C c+\frac{u}{\sqrt{\chi_{2}^{1}}}\left(\phi\left(m_{1}\right)-\phi\left(n_{1}\right)\right)+\frac{1-u}{\sqrt{\chi_{2}^{2}}}\left(\phi\left(m_{2}\right)-\phi\left(n_{2}\right)\right)}{A}
\end{aligned}
$$

When $\theta_{2}=\bar{\theta}_{1}-\epsilon$, we suppose that the equilibrium price is $\bar{\theta}_{1}-\zeta$, then we show that $\bar{\theta}_{1}-\zeta$ and $\bar{\theta}_{1}-\epsilon$ verify the equilibrium price equation. In this case 
of negative common error,

$$
\begin{aligned}
m_{i}^{\prime} & =\sqrt{\chi_{2}^{i}}\left(\widehat{\rho}_{1}\left(\bar{\theta}_{1}-\zeta+c-\bar{\theta}_{1}\right)+\rho_{2}^{s i}\left(\bar{\theta}_{1}-\zeta+c-\bar{\theta}_{1}+\epsilon\right)\right) \\
& =\sqrt{\chi_{2}^{i}}\left(\widehat{\rho}_{1}(-\zeta+c)+\rho_{2}^{s i}(-\zeta+c+\epsilon)\right) \\
& =-n_{i} \\
n_{i}^{\prime} & =\sqrt{\chi_{2}^{i}}\left(\widehat{\rho}_{1}\left(\bar{\theta}_{1}-\zeta-c-\bar{\theta}_{1}\right)+\rho_{2}^{s i}\left(\bar{\theta}_{1}-\zeta-c-\bar{\theta}_{1}+\epsilon\right)\right) \\
& =\sqrt{\chi_{2}^{i}}\left(\widehat{\rho}_{1}(-\zeta-c)+\rho_{2}^{s i}(-\zeta-c+\epsilon)\right) \\
& =-m_{i}
\end{aligned}
$$

and

$$
\begin{aligned}
p_{2}^{\bar{\theta}_{1}-\epsilon}-\bar{\theta}_{1} & =p_{2}^{\bar{\theta}_{1}-\epsilon}-\frac{A^{\prime}}{A^{\prime}} \bar{\theta}_{1} \\
& =\frac{-C^{\prime} c+\frac{u}{\sqrt{\chi_{2}^{1}}}\left(\phi\left(m_{1}^{\prime}\right)-\phi\left(n_{1}^{\prime}\right)\right)+\frac{1-u}{\sqrt{\chi_{2}^{2}}}\left(\phi\left(m_{2}^{\prime}\right)-\phi\left(n_{2}^{\prime}\right)\right)}{A^{\prime}}
\end{aligned}
$$

It is easy to show that $A^{\prime}=A, B^{\prime}=B$, and $C^{\prime}=-C$, then we have

$$
\begin{aligned}
p_{2}^{\bar{\theta}_{1}-\epsilon}-\bar{\theta}_{1} & =\frac{C c+\frac{u}{\sqrt{\chi_{2}^{1}}}\left(\phi\left(n_{1}\right)-\phi\left(m_{1}\right)\right)+\frac{1-u}{\sqrt{\chi_{2}^{2}}}\left(\phi\left(n_{2}\right)-\phi\left(m_{2}\right)\right)}{A^{\prime}} \\
& =-\zeta
\end{aligned}
$$

For the second part, because

$$
\begin{aligned}
\operatorname{Var}\left(\frac{p_{2}^{c}-p_{1}}{p_{1}} \mid \bar{\theta}_{1}, p_{1}=\theta_{1}\right)= & E\left(\left(\frac{p_{2}^{c}-p_{1}}{p_{1}} \mid \bar{\theta}_{1}, p_{1}=\theta_{1}\right)^{2}\right) \\
& -\left(E\left(\frac{p_{2}^{c}-p_{1}}{p_{1}} \mid \bar{\theta}_{1}, p_{1}=\theta_{1}\right)\right)^{2} \\
\operatorname{Var}\left(\frac{p_{2}^{n}-p_{1}}{p_{1}} \mid \bar{\theta}_{1}, p_{1}=\theta_{1}\right)= & E\left(\left(\frac{p_{2}^{n}-p_{1}}{p_{1}} \mid \bar{\theta}_{1}, p_{1}=\theta_{1}\right)^{2}\right) \\
& -\left(E\left(\frac{p_{2}^{n}-p_{1}}{p_{1}} \mid \bar{\theta}_{1}, p_{1}=\theta_{1}\right)\right)^{2} \\
E\left(p_{2}^{c} \mid \bar{\theta}_{1}, p_{1}=\theta_{1}\right)= & E\left(p_{2}^{n} \mid \bar{\theta}_{1}, p_{1}=\theta_{1}\right)
\end{aligned}
$$

we only need compare $E\left(\left(\frac{p_{2}^{c}-p_{1}}{p_{1}} \mid \bar{\theta}_{1}, p_{1}=\theta_{1}\right)^{2}\right)$ and $E\left(\left(\frac{p_{2}^{n}-p_{1}}{p_{1}} \mid \bar{\theta}_{1}, p_{1}=\theta_{1}\right)^{2}\right)$.

Before comparison, we first calculate the partial derivative of $m_{i}$ with respect 
to $\theta_{2}$

$$
\begin{aligned}
\frac{\partial m_{1}}{\partial \theta_{2}} & =\sqrt{\chi_{2}^{2}}\left(\left(\widehat{\rho}_{1}+\rho_{2}^{s 1}\right) \frac{\partial p_{2}}{\partial \theta_{2}}-\rho_{2}^{s 1}\right) \\
& =\sqrt{\chi_{2}^{2}}\left(\left(\widehat{\rho}_{1}+\rho_{2}^{s 1}\right) \frac{B}{A}-\rho_{2}^{s 1}\right) \\
& =\frac{\sqrt{\chi_{2}^{2}} \widehat{\rho}_{1}}{A}(1-u)\left(1-\Phi\left(m_{2}\right)+\Phi\left(n_{2}\right)\right)\left(\rho_{2}^{s 2}-\rho_{2}^{s 1}\right) \\
< & 0 \\
\frac{\partial m_{2}}{\partial \theta_{2}} & =\sqrt{\chi_{2}^{2}}\left(\left(\widehat{\rho}_{1}+\rho_{2}^{s 2}\right) \frac{\partial p_{2}}{\partial \theta_{2}}-\rho_{2}^{s 2}\right) \\
& =\frac{\sqrt{\chi_{2}^{2}} \widehat{\rho}_{1}}{A} u\left(1-\Phi\left(m_{1}\right)+\Phi\left(n_{1}\right)\right)\left(\rho_{2}^{s 1}-\rho_{2}^{s 2}\right) \\
& >0
\end{aligned}
$$

When $\theta_{2}=\bar{\theta}_{1}, p_{2}=\bar{\theta}_{1}, m_{i}=n_{i}$ and $1-\Phi\left(m_{i}\right)-\Phi\left(n_{i}\right)=0$. Then

$$
\begin{aligned}
& 1-\Phi\left(m_{2}\right)-\Phi\left(n_{2}\right)>0,1-\Phi\left(m_{1}\right)-\Phi\left(n_{1}\right)<0 \text { when } \theta_{2}<\bar{\theta}_{1} \\
& 1-\Phi\left(m_{2}\right)-\Phi\left(n_{2}\right)<0,1-\Phi\left(m_{1}\right)-\Phi\left(n_{1}\right)>0 \text { when } \theta_{2}>\bar{\theta}_{1}
\end{aligned}
$$

From the relation above, we have

$$
\begin{aligned}
& u<\frac{1}{1-\frac{\left(\widehat{\rho}_{1}+\rho_{2}^{s 1}\right)\left(1-\Phi\left(m_{1}\right)-\Phi\left(n_{1}\right)\right)}{\left(\widehat{\rho}_{1}+\rho_{2}^{s 2}\right)\left(1-\Phi\left(m_{2}\right)-\Phi\left(n_{2}\right)\right)}} \\
& -u\left(\widehat{\rho}_{1}+\rho_{2}^{s 1}\right)\left(1-\Phi\left(m_{1}\right)-\Phi\left(n_{1}\right)\right) \\
& \Leftrightarrow \quad<(1-u)\left(\widehat{\rho}_{1}+\rho_{2}^{s 2}\right)\left(1-\Phi\left(m_{2}\right)-\Phi\left(n_{2}\right)\right) \quad \text { when } \theta_{2}<\bar{\theta}_{1} \\
& -u\left(\widehat{\rho}_{1}+\rho_{2}^{s 1}\right)\left(1-\Phi\left(m_{1}\right)-\Phi\left(n_{1}\right)\right) \quad \text { when } \theta_{2}>\bar{\theta}_{1} \\
& >(1-u)\left(\widehat{\rho}_{1}+\rho_{2}^{s 2}\right)\left(1-\Phi\left(m_{2}\right)-\Phi\left(n_{2}\right)\right) \\
& \Leftrightarrow \quad-\frac{C}{A}<0 \quad \text { when } \theta_{2}<\bar{\theta}_{1}
\end{aligned}
$$

We have shown in the proof of Proposition 8 that $\frac{\partial p_{2}}{\partial c}=-\frac{C}{A}$. Then

$$
\begin{aligned}
& p_{2}^{c}<p_{2}^{n} \quad \text { when } \theta_{2}<\bar{\theta}_{1} \\
& p_{2}^{c}>p_{2}^{n} \text { when } \theta_{2}>\bar{\theta}_{1}
\end{aligned}
$$

The last step is to show $E\left(\left(\frac{p_{2}^{c}-p_{1}}{p_{1}} \mid \bar{\theta}_{1}, p_{1}=\theta_{1}\right)^{2}\right)>E\left(\left(\frac{p_{2}^{n}-p_{1}}{p_{1}} \mid \bar{\theta}_{1}, p_{1}=\theta_{1}\right)^{2}\right)$ 
which is trivial.

\section{References}

Amihud, Y. \& Mendelson, H. 1986. Liquidity and stock returns, Financial Analysts Journal 42: 43-48.

Barron, O. \& Karpoff, J. 2004. Information precision, transaction costs, and trading volume, Journal of Banking and Finance 28: 1207-1223.

Blume, L. \& Easley, D. 1984. Rational expectations equilibrium: An alternative apporach, Journal of Economic Theory 34: 116-129.

Blume, L., Easley, D. \& O'Hara, M. 1994. Market statistics and technical analysis: The role of volume, Journal of Finance 49: 153-181.

Boudoukh, J., Richardson, M. \& Whitelaw, R. 1994. A tale of three schools: Insights on autocorrelation of short-horizon stock returns, Review of Financial Studies 7: 45-67.

Brennan, M. \& Chordia, T. 1993. Brokerage commission schedules, Journal of Finance 48: 1379-1402.

Brown, D. \& Jenning, R. 1989. On technical analysis, Review of Financial Studies 4: 527-551.

Cheng, H. 2005a. Trading volume, asset price and transaction costs, Working Paper.

Constantinides, G. 1986. Capital market equilibrium with transaction costs, Journal of Political Economy 94: 842-862.

Green, C., Maggioni, P. \& Murinde, V. 2000. Regulatory lessons for emerging stock markets from a century of evidence on transactions costs and share price volatility in the london stock exchange, Journal of Banking and Finance 24: 577-601.

Hau, H. 2006. The role of transaction costs for financial volatility: Evidence from the paris bourse, Journal of European Economic Association, Forthcoming 
Heaton, J. \& Lucas, D. 1996. Evaluating the effects of incomplete markets on risk sharing and asset pricing, Journal of Political Economy 104: 443-487.

Hellwig, M. 1982. Rational expectations equilibrium with conditioning on past prices: A mean-variance example, Journal of Economic Thoery 26: 279312 .

Huang, M. 2003. Liquidity shocks and equilibrium liquidity premia, Journal of Economic Theory 103: 104-129.

Jones, C. \& Seguin, P. 1997. Transaction costs and prices volatility: Evidence from commission deregulation, American Economic Review 87: 728-737.

Lo, A., Mamaysky, H. \& Wang, J. 2004. Asset prices and trading volume: Under fixed transaction costs, Journal of Political Economy 112: 1054-1090.

Lo, A. \& Wang, J. 2000. Trading volume: Definition, data analysis, and implications of portfolio theory, Review of financial stuides 13: 257-300.

Stiglitz, J. 1989. Using tax policy to curb speculative short-term trading, Journal of Financial Servies Research 3: 101-115.

Summers, L. \& Summers, V. 1989. When financial markets work too well: A cautious case for a securities transactions tax, Journal of Financial Services Research 3: 261-286.

Umlauf, S. 1993. Transaction taxes and the behavior of the swedish stock market, Journal of Financial Economics 33: 227-240.

Vayanos, D. 1998. Transaction costs and asset prces: A dynamic equilibrium model, Review of Financial Studies 11: 1-58. 